\title{
RPA-Approach to the Excitations of the Nucleon, Part II: Phenomenology
}

\author{
S. Hardt, J. Geiss, H. Lenske and U. Mosel \\ Institut für Theoretische Physik \\ Universität Giessen \\ Heinrich-Buff-Ring 16 \\ D-35392 Giessen, Germany
}

October 6, 2018

\begin{abstract}
The tensor-RPA approach developed previously in part I is applied to the NambuJona-Lasinio (NJL) model. As a first step we investigate the structure of DiracHartree-Fock solutions for a rotationally and isospin invariant ground-state density. Whereas vacuum properties can be reproduced, no solitonic configuration for a system with unit baryon number is found. We then solve the tensor-RPA equation employing simple models of the nucleon ground state. In general the $p h$ interaction effects a decrease of the excited states to lower energies. Due to an enhanced level density at low energies the obtained spectra cannot be matched with the experimental data when a standard MIT-bag configuration is used. However, when the size of the nucleon quark core is reduced to $\approx 0.3 \mathrm{fm}$ a fair description of the baryon spectrum in the positive-parity channel is achieved. For this purpose the residual interaction turns out to be crucial and leads to a significant improvement compared with the mean-field spectra.
\end{abstract}

\section{Introduction}

In a previous paper [1] we have developed a method to compute the excited states of the nucleon in the framework of a Dirac-RPA description applied to an effective quark model. The method is based on the tensor coupling of particle-hole $(p h)$ configurations on the nucleon ground state and allows to construct excited states with good angular momentum $(J)$ and isospin $(T)$ quantum numbers.

In the present work we apply the tensor-RPA formalism to a simple quark model of Nambu-Jona-Lasinio (NJL) type. Our investigations are analogous to extensions of the NJL soliton model beyond the mean-field level [2], with two major differences.

First of all we do not use an Euclidean path integral formalism to derive many-body 
approximation schemes, but rather rely on the Dirac-RPA method developed in part I of this paper. This approach is formally more similar to the conventional formulation of nonrelativistic many-body theory. As a consequence, we are spared the problem of continuing back the vector potentials to Minkowski space which has turned out to be a quite nontrivial task [3, 4].

Secondly we treat the constraints set by the symmetries of the interaction in a stricter way than it is done in the NJL soliton model. In the latter states with good $J / T$ quantum numbers are projected out from hedgehog configurations by perturbative cranking 《, 5. In contrast to that we construct a ground-state density matrix which is invariant with respect to rotations in coordinate and isospin space. In this way we obtain a spectrum of single-particle states carrying good $J / T$ quantum numbers which can be used to build collective baryonic states by means of the tensor-RPA techniques.

The strict treatment of symmetries has important implications for the structure of the mean field. Whereas in a simple version of the NJL soliton model the scalar field is accompanied by a pseudoscalar mode [4], the ground state configuration of our model does not allow for such a mode. Without the pseudoscalar field the ground state as a self-consistent solution of a regularized Hartree-Fock equation turns out to be an unbound system of quarks.

An issue to be addressed in the context of a model for the excited states of the nucleon is the question of confinement. Due to the lack of a confinement mechanism unphysical single-quark thresholds appear in the NJL soliton model [2] as well as in NJL-based approaches relying on the Bethe-Salpeter equation in the quark-quark [6] or quark-antiquark channel [7]. In the NJL soliton model all excited single-quark states are unbound. While this might be no serious drawback as long as we consider the nucleon ground state, the description of the excitation spectrum becomes questionable in such a description.

There exist models for baryons [8] as well as for mesons [9] which incorporate the confinement property of QCD, but the origin of these models lies in a nonrelativistic potential picture for the two-body interaction. In that framework it seems impossible to describe the structure of the QCD vacuum and the spontaneous breakdown of chiral symmetry. To our knowledge so far nobody has formulated a consistent theory based on quark degrees of freedom exclusively which confines a system of three quarks on mean-field level and models the quark sector of the QCD vacuum at the same time. However, at the price of introducing boson fields that couple to the quarks this goal can be reached, like in the chromodielectric model [10]. A few attempts towards a unified description of vacuum properties and quark confinement have been made [11, 12, 13], but still these models suffer from a number of drawbacks. To give an example, an interaction of the form $\delta^{(4)}(p)$ as in Ref. 111 incorporates confinement in the sense of a pole-free quark propagator, but cannot be consistently used for finite systems as the nucleon. Recently a confining model has been proposed which contains a remnant of the functional integration over gluon degrees of freedom by a statistical treatment of the 4-quark vertex [14]. However, the applicability of this model to baryonic systems is yet to be proven.

For applying the tensor-RPA scheme it is necessary to model the nucleon by a mean-field configuration from which at least some gross properties of the excitation spectra, e. g. the 
absence of free quarks, can be derived. With no suitable mechanism for dynamical quark confinement at hand we have to restrict ourselves to a simplified description of the ground state. For this purpose we use a phenomenolocigal basis of single-particle wavefunctions taken from a bag-model together with a residual interaction of NJL type. The bag model clearly does not represent a self-consistent configuration in such a framework but provides us with a simple way to incorporate confinement in our description.

The present work is divided into two major sections.

In section 2 we will first discuss self-consistent mean-field configurations of the vacuum. We further investigate the structure of the solutions when we add three valence quarks to the system. These investigations within the NJL model are based on the most general form of the self-energy compatible with the symmetries of the ground state. Subsequently we briefly discuss a class of phenomenological models for the nucleon.

In section 3 we focus our attention on the excitation spectrum as obtained with the tensorRPA method. We compare different models for the ground state as well as different forms of the residual interaction. We discuss the effects of a pion-exchange interaction which was recently claimed to be of special importance for the baryon spectrum [15]. Using a local color-current interaction and reducing the size of the quark core to $\approx 0.3 \mathrm{fm}$ the excitation spectrum in the positive-parity channel can be reproduced fairly well.

\section{The Nucleon Ground State}

The major part of this section is devoted to an investigation of self-consistent solutions of the NJL model on mean-field level. Solitonic field configurations of the NJL model in the sector with unit baryon number $(B=1)$ have been studied extensively in the past [4 $[5]$, but the prescription used in these studies to project on physical states with good angular momentum $(\mathrm{J})$ and isospin $(\mathrm{T})$ is rather crude. The tensor-RPA formalism developed in part I constitutes a powerful tool to investigate the excitation spectrum of the nucleon in any $J / T$ channel. It is therefore worthwhile to explore how far the NJL model is suited to provide a ground-state configuration for the tensor RPA.

We will find that in a model space of MIT-bag states with an appropriately large bag radius vacuum properties as obtained from the gap equation can be reproduced with high accuracy, but the $B=1$ system does not emerge as a bound solitonic object.

With focus on a description of the excitation spectrum we proceed to discuss a class of simple models for the nucleon ground state, thus giving up self-consistency but introducing quark confinement on a phenomenological level.

\subsection{Self-Consistent Mean-Field Configurations}

As a model for low-energy QCD we consider the well known NJL Lagrangian restricted to flavor $S U(2)$ (see, e. g., [16])

$$
\mathcal{L}=\bar{\Psi}\left(i \not \partial-\hat{m}_{0}\right) \Psi-G j_{a \mu} j_{a}^{\mu},
$$


where a color-current interaction with

$$
j_{a}^{\mu}=\bar{\Psi} \frac{\lambda_{a}}{2} \gamma^{\mu} \Psi
$$

is used. We have introduced a $S U(2)$ current-quark mass matrix $\hat{m}_{0}$ and denoted the generators of the color group by $\frac{\lambda_{a}}{2}$. The model defined in Eq. (I) is chirally invariant in case of a vanishing mass matrix $\hat{m}_{0}$.

To compute exchange terms we take the Fierz transform of the color-current interaction in Eq. (1) and obtain

$$
\begin{aligned}
\mathcal{F}\left[G\left(\bar{\Psi} \gamma_{\mu} \frac{\lambda_{a}}{2} \Psi\right)^{2}\right] \\
=\frac{G_{s}}{9}\left[-(\bar{\Psi} \Psi)^{2}+\left(\bar{\Psi} \gamma_{5} \Psi\right)^{2}-(\bar{\Psi} \tau \Psi)^{2}+\left(\bar{\Psi} \gamma_{5} \tau \Psi\right)^{2}\right] \\
\quad+\frac{G_{v}}{9}\left[\left(\bar{\Psi} \gamma_{\mu} \Psi\right)^{2}-\left(\bar{\Psi} \gamma_{5} \gamma_{\mu} \Psi\right)^{2}+\left(\bar{\Psi} \gamma_{\mu} \tau \Psi\right)^{2}-\left(\bar{\Psi} \gamma_{5} \gamma_{\mu} \tau \Psi\right)^{2}\right]+\text { c.o. }
\end{aligned}
$$

with

$$
G_{v}=\frac{G_{s}}{2}=G .
$$

The color octet terms, indicated by c.o., are not displayed, because they do not play any role for matrix elements taken between color-singlet states which are the only terms of interest. The evaluation of exchange terms of the NJL-interaction is done by computing the direct contribution of the Fierz transform. The interaction of Eq. (3i) is only used in the direct channel and clearly exhibits the Lorentz and isospin structure of the exchange matrix elements.

Chiral invariance is preserved if we treat $G_{s}$ and $G_{v}$ as independent parameters, so in the following we relax the condition in Eq. (4) and consider a more general class of models with $G_{s} \neq 2 G_{v}$. There are some attempts to fix the ratio $\frac{G_{v}}{G_{s}}$ from phenomenology [17, 18], but so far the results are contradictory.

\subsubsection{The $B=0$ vacuum sector}

First we investigate the vacuum-structure obtained from the NJL model on mean-field level. Starting from the color-current interaction Eq. (1) the Hartree contribution to the self-energy vanishes for a color-singlet vacuum. The Fock term is computed by evaluating the direct contribution from the Fierz transform. Assuming a Poincaré-invariant vacuum, the only nonvanishing contribution to the self-energy is a scalar term

$$
\Sigma_{s}=-\frac{2}{9} G_{s}\langle\bar{\Psi} \Psi\rangle .
$$

The scalar self-energy, which is up to a factor the chiral condensate of the QCD vacuum, can be computed by solving a gap equation, as first shown by Y. Nambu and G. Jona- 
Lasinio in 1961 [19]. For flavor $S U(2)$ the chiral condensate is given by

$$
\langle\bar{\Psi} \Psi\rangle=-\frac{6}{\pi^{2}} \int_{m}^{\infty} d E \sqrt{E^{2}-m^{2}} m R^{2}[E]
$$

with

$$
m=m_{0}+\Sigma_{s}, \quad m_{u}=m_{d}=m_{0} .
$$

The cut-off function $R$ is chosen as in part I. With the expression for the chiral condensate Eq. (5) represents a nonlinear equation for the scalar self energy, which can be solved iteratively.

We take up the problem of vacuum structure in the NJL model and tackle it from a different angle. The Dirac Hartree-Fock problem for the nucleon will be solved in a basis of MIT-bag states, therefore it is advisable to first perform the corresponding calculation for the vacuum in the same basis. By comparing the results with the solution of the gap equation we get an estimate on the importance of finite-size effects in a basis corresponding to a certain bag radius. Furthermore it allows us to make a statement about the stability of a translationally invariant vacuum state, which enters the gap equation Eq. (5) only as an assumption.

Let us consider the mean-field potential of the vacuum in a similar way as the corresponding expression for the nucleon we have given in part I of this paper. From the Fierz transform Eq. (3) we obtain the Lorentz-scalar contribution to the mean field as

$$
V_{M F}^{s}=-\frac{2}{9} G_{s} \sum_{p q i}\left(\int d^{3} r \bar{\Psi}_{p} \Psi_{q} \bar{\Psi}_{i} \Psi_{i}\right) a_{p}^{\dagger} a_{q} R^{2}\left[\epsilon_{i}\right] \theta\left(-\epsilon_{i}\right) .
$$

The time-like component of the Lorentz-vector self-energy reads

$$
V_{M F}^{v}=\frac{2}{9} G_{v} \sum_{p q i}\left(\int d^{3} r \Psi_{p}^{\dagger} \Psi_{q} \Psi_{i}^{\dagger} \Psi_{i}\right) a_{p}^{\dagger} a_{q} R^{2}\left[\epsilon_{i}\right] \theta\left(-\epsilon_{i}\right) .
$$

The sums in these expressions are over the self-consistent one-body states. The singleparticle energies $\epsilon_{i}$ are the eigenvalues of the mean-field Hamiltonian including the kineticenergy contribution. The form of the regularization function $R$ is yet to be specified.

Since the vacuum is a $\left(J^{p}=0^{+}, T=0\right)$ state, there are no contributions to the mean field from isovector, pseudoscalar, 3-vector or axial-vector terms. A nonvanishing time-like component of a four-vector breaks Lorentz invariance. Hence the potential in Eq. (9) should have no influence on the dynamics of the system. However, besides the scalar condensate the vector potential is the only nonvanishing contribution to the mean field in the corresponding problem for the nucleon. Therefore, by inclusion of the vector potential in the vacuum calculation we study the fluctuations of the baryon density while keeping the effective baryon number zero by means of a Lagrange multiplier.

For this purpose we consider the constrained Hartree-Fock problem

$$
V_{M F}^{v} \longrightarrow V_{M F}^{v}-\lambda \frac{2}{9} G_{v} B,
$$


where the baryon number operator is given by

$$
B=\frac{1}{3} \sum_{p q}\left(\int d^{3} r \Psi_{p}^{\dagger} \Psi_{q}\right) a_{p}^{\dagger} a_{q}
$$

The Lagrange multiplier $\lambda$ is determined such that the effective baryon number

$$
\frac{1}{3}\left(\int d^{3} r\left(\sum_{i} \Psi_{i}^{\dagger} \Psi_{i} R^{2}\left[\epsilon_{i}\right] \theta\left(-\epsilon_{i}\right)\right)-\frac{1}{3} \lambda\right)
$$

vanishes.

The expressions for the mean-field potential, Eqs. (8) and (9), have been given in the self-consistent basis where only the diagonal elements of the regularized mean-field Hamiltonian enter as an argument of the cut-off function $R$. In a practical calculation we start with a field configuration in the vicinity of the self-consistent solution, but according to the prescription for the Hartree-Fock iteration scheme given in part I of this paper we assume that the off-diagonal elements of the mean-field Hamiltonian can be neglected in $R$, so that the operators we have to evaluate are of the same structure as above.

Owing to the symmetry of the vacuum the single-particle wavefunctions can be written as

$$
\Psi_{p}=\left(\begin{array}{c}
g_{p} \chi_{\kappa_{p}}^{\mu_{p}} \\
-i f_{p} \chi_{-\kappa_{p}}^{\mu_{p}}
\end{array}\right)
$$

where $\chi_{\kappa}^{\mu}$ are the spin-angle functions (see, e. g., [20]) with angular-momentum projection $\mu$. $\kappa$ denotes the eigenvalue of $K=\beta(\boldsymbol{\Sigma} \cdot \mathbf{l}+1)$. The radial functions $g, f$ are to be determined self-consistently.

To solve for the self-consistent states, we write the mean-field Hamiltonian in a basis of eigenmodes of a spherical cavity with radius $R_{c}$

$$
\Psi=N\left(\begin{array}{c}
j_{l}(p r) \chi_{\kappa}^{\mu} \\
i \frac{p}{E+m} \operatorname{sgn}(\kappa) j_{\bar{l}}(p r) \chi_{-\kappa}^{\mu}
\end{array}\right),
$$

where $m$ is the quark mass and $E=\sqrt{\mathbf{p}^{2}+m^{2}}$. The eigenvalues $p$ are fixed by the boundary condition. In general the structure of the field configuration which can be represented with a set of basis states depends on the boundary condition imposed on these states [21]. We consider the two different forms

1. MIT-bag boundary condition

$$
\left.\mathbf{n} \cdot \mathbf{J}\right|_{R_{c}}=0,
$$

where $\mathbf{n}$ is a unit vector normal to the bag surface and $\mathbf{J}=\bar{\Psi} \gamma \Psi$.

2. nonrelativistic boundary condition

$$
j_{l}\left(p R_{c}\right)=0 .
$$


Both conditions lead to a set of orthogonal basis states.

For the mass parameter $m$ we choose a value consistent with the solution of the gap equation Eq. (5), where $m_{0}=5 \mathrm{MeV}$. A set of eigenmodes of the spherical cavity with a mass parameter equal to the constituent-quark mass from the gap equation also serves as an initialization for our iteration scheme.

We consider two different forms for the cut-off function $R$ :

1. Woods-Saxon cut-off

$$
R^{2}[x]=\left(1+e^{-\frac{x+\Lambda}{\sigma}}\right)^{-1}
$$

2. Proper-time cut-off

$$
R^{2}[x]=\left\{\begin{array}{cl}
1 & : \quad x \geq 0 \\
\operatorname{erfc}\left(\left|\frac{x}{\Lambda}\right|\right) & : \quad x<0
\end{array} .\right.
$$

The Woods-Saxon parametrization is used to approximate a step-function $\theta(x+\Lambda)$. However, for very small width parameters $\sigma$ the iteration scheme fails to converge. Thus for reasons of numerical stability we choose $\sigma=10 \mathrm{MeV}$.

The proper-time cut-off is the standard scheme used in the NJL soliton model [4, 5]. The corresponding form of $R$ can be derived by writing the fermion determinant appearing in the bosonized NJL action as a proper-time integral [4, 5].

In the iteration scheme we successively solve for the eigenvalues and eigenvectors of the mean-field Hamiltonian' $H_{M F}^{(i)}$ corresponding to step $i$ of the iteration process. The matrix to be diagonalized splits up in several submatrices in the spaces of states with the same angular-momentum/parity $(J / P)$ quantum numbers. Typically we work in a cavity with $R_{c}=8 \mathrm{fm}$. The basis space usually contains $4000-5000$ states, with an equal number of such with positive and negative energy. The usual iteration scheme, where $H_{M F}^{(i)}$ is defined with the eigenvectors of step $i-1$ is found to converge very slowly. Convergence can be decisively improved by application of a modified scheme:

$$
H_{M F}^{(i)}=H_{M F}\left(\frac{1}{i-k(i)} \sum_{n=k(i)+1}^{i} \rho^{(n)}\right)
$$

with

$$
k(i)=k_{0} \operatorname{int}\left(\frac{i-1}{k_{0}}\right) .
$$

$\rho^{(n)}$ is the approximation for the ground-state density matrix in step $n$. The initial guess $\rho^{(1)}$ should not be too far away from the self-consistent solution. The essence of the modified iteration scheme is an averaging over the density matrices of several iteration steps which is reset after $k_{0}$ steps. For $k_{0}=10$ convergence is usually achieved after $20-30$ steps.

Comparing the results of these calculations with the solution of the gap equation we find a very good overall agreement. In Fig. 1 we show the chiral condensate $-\langle\bar{\Psi} \Psi\rangle$ as a function of $r$ for different boundary conditions and cut-off schemes. The calculations

\footnotetext{
${ }^{1}$ In the notation of part I of this paper the regularized mean-field Hamiltonian carries a prime which we leave away in the following
} 


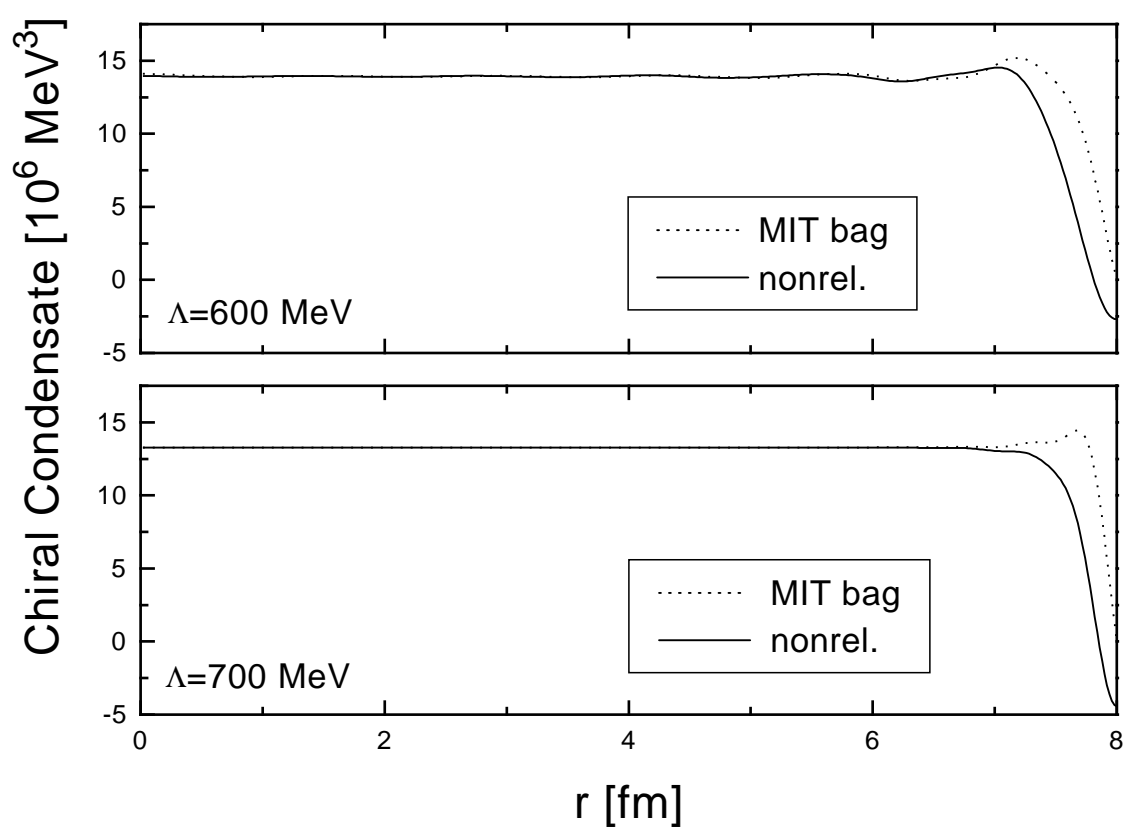

Figure 1: $\quad-\langle\bar{\Psi} \Psi\rangle$ for the Woods-Saxon (above) and the proper-time (below) cut-off scheme. In both cases $\Lambda$ and $G_{s}$ were fixed to produce a constituent-quark mass of $400 \mathrm{MeV}$. Different boundary conditions are compared.

were done with $G_{v}=0$. In both cut-off schemes the values for the condensates we obtain from the gap equation (Woods-Saxon: $1.39 \cdot 10^{7} \mathrm{MeV}^{3}$, proper-time: $1.33 \cdot 10^{7} \mathrm{MeV}^{3}$ ) are reproduced very well. The boundary condition has an effect on the structure of the vacuum only in the vicinity of the bag boundary. When the dimension of the basis space is increased from 4000 to 6000 , the results are left unchanged within the line width of the graphical representation.

The results show that a translationally invariant condensate in fact solves the DysonSchwinger equation in the vacuum sector. In the gap equation translational invariance enters as an a priori assumption which, however, seems to have been justified by the results displayed in Fig. 1.

When we switch on the vector potential and solve the constraint Hartree-Fock problem Eq. (10) we observe that the chiral condensate slightly increases (i. e. $\langle\bar{\Psi} \Psi\rangle$ increases in magnitude), as displayed in Fig. 2. For these calculations the proper-time cut-off was chosen and the constituent-quark mass was fixed to a value $m=600 \mathrm{MeV}$. If not stated otherwise, we use the nonrelativistic boundary condition in our calculations. The coupling constant in the vector channel was adjusted to the value obtained from the color-current interaction $G_{v}=G_{s} / 2$.

Without any vector potential the chiral condensate as obtained from the gap equation $\left(1.33 \cdot 10^{7} \mathrm{MeV}^{3}\right)$ is reproduced with high accuracy even in a smaller cavity with $R_{c}=4 \mathrm{fm}$. When we increase the cavity radius to $8 \mathrm{fm}$ the shift of the condensate effected by the vector potential is less pronounced than in the small cavity. We thus conclude that the shift will vanish in the continuum limit. Whereas the net baryon number can be fixed to zero with the help of a Lagrange multiplier, the net baryon density will not be a completely smooth function due to constraints set by the boundary condition. The fact that 


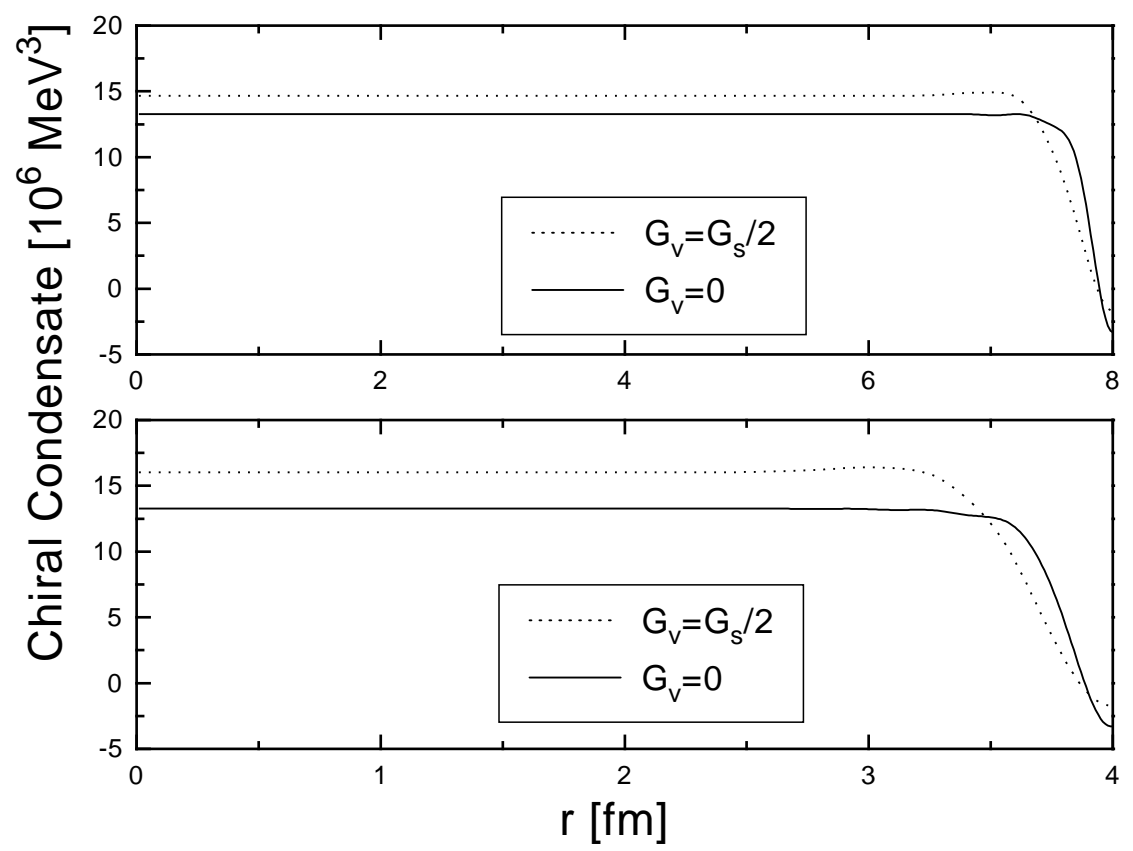

Figure 2: $-\langle\bar{\Psi} \Psi\rangle$ in a cavity with $R_{c}=8 \mathrm{fm}$ (above) and $R_{c}=4 \mathrm{fm}$ (below)

the vector potential couples to the baryon density offers an explanation for the induced effects which seem to vanish in the continuum limit.

\subsection{2 $B=1$-configurations}

We now turn to self-consistent configurations with unit baryon number. For the computation of $B=1$ configurations we use a similar prescription as for the vacuum, but we add three valence quarks to the system. This amounts to replacing the vacuum occupation factors $\theta\left(-\epsilon_{i}\right)$ by $\bar{\rho}(i)$ in each expression. The density matrix $\bar{\rho}$, which is averaged over the $J / T$ projection quantum numbers of the valence quark configuration, was defined in part I. By such an averaging procedure we neglect the nonscalar parts of the ground-state density.

Properties of the solutions are always extracted from the difference between the results for the $B=1$ and the $B=0$ system, which is the vacuum by definition. Effectively, this means that all additive quantum numbers of the vacuum are renormalized to zero. Still, vacuum polarization effects are fully accounted for.

In such a prescription the baryon number is given by

$$
B=\frac{1}{3} \int d^{3} r\left(\sum_{i^{\prime}} \Psi_{i^{\prime}}^{\dagger} \Psi_{i^{\prime}} R^{\prime 2}\left[\epsilon_{i^{\prime}}\right] \bar{\rho}\left(i^{\prime}\right)-\sum_{i} \Psi_{i}^{\dagger} \Psi_{i} R^{2}\left[\epsilon_{i}\right] \theta\left(-\epsilon_{i}\right)\right) .
$$

The self-consistent single-particle states of the $B=1$ system are indicated by primed labels. Note that due to vacuum polarization effects the baryon number contribution of the Dirac sea might be different in the calculations with and without valence quarks. In order to fix the baryon number difference Eq. (21) to one, two slightly different cut-off 
functions $R^{\prime}$ and $R$ were chosen by readjusting the cut-off scale $\Lambda$. The difference of the original and the readjusted cut-off scale was always found to be less than $1 \mathrm{MeV}$. If the same cut-off is used in the two calculations, the resulting field configurations look very much the same as the configurations with readjusted cut-off, but the total energy of the system including valence quarks is found to be lower than the vacuum energy. The energy is defined as the expectation value of the NJL Hamiltonian in a ground state with regularized one-body density

$$
E=\sum_{i^{\prime}} \epsilon_{i^{\prime}} \bar{\rho}\left(i^{\prime}\right) R^{\prime 2}\left[\epsilon_{i^{\prime}}\right]-\frac{1}{2} \sum_{i^{\prime} j^{\prime}}\left\langle i^{\prime} j^{\prime}\left|\bar{v}_{N J L}\right| i^{\prime} j^{\prime}\right\rangle \bar{\rho}\left(i^{\prime}\right) \bar{\rho}\left(j^{\prime}\right) R^{\prime 2}\left[\epsilon_{i^{\prime}}\right] R^{\prime 2}\left[\epsilon_{j^{\prime}}\right]
$$

where the two-body interaction is derived from the NJL Lagrangian Eq. (3) and given by

$$
\left\langle i j\left|v_{N J L}\right| i j\right\rangle=G \int d^{3} r \bar{\Psi}_{i} \gamma_{\mu} \frac{\lambda_{a}}{2} \Psi_{i} \bar{\Psi}_{j} \gamma^{\mu} \frac{\lambda_{a}}{2} \Psi_{j}
$$

When we include the vector part of the self energy, we subtract the baryon density as obtained from the vacuum calculation and define the remaining contribution as the effective baryon density entering the equations as a source term for the vector potential. In the continuum limit, where boundary effects become unimportant, this corresponds to the introduction of a Lagrange multiplier, similar to Eq. (10). In all the calculations for the $B=1$ system the proper-time cut-off was used.

In Fig. 3 the baryon density is displayed for different constituent-quark masses and $G_{v}=0$. The coupling constant in the scalar channel was fixed to reproduce phenomenologically reasonable values for the chiral condensate of the order of $(-240 \mathrm{MeV})^{3} \approx 1.4 \cdot 10^{7} \mathrm{MeV}^{3}$. The self-consistent field configurations are spread out over the entire cavity. The structure of the solutions changes very little when the constituent-quark mass is varied. Obviously the scalar field alone does not provide enough attraction to form a bound, solitonic object.

From the NJL soliton model it is known that below a critical constituent-quark mass no solitons are found [4. Since the density profile is invariant under variations of the constituent-quark mass in the region below $1 \mathrm{GeV}$, we do not expect that such a phase transition occurs in our model for any constituent-quark masses up to a few $G e V$.

Due to vacuum polarization effects the baryon density becomes negative in the vicinity of the bag boundary. The masses of the configurations are $3769 \mathrm{MeV}, 4586 \mathrm{MeV}$ and $5440 \mathrm{MeV}$ for $\mathrm{m}$ equal to $400 \mathrm{MeV}, 600 \mathrm{MeV}$ and $800 \mathrm{MeV}$, respectively. The large values for the masses indicate that the vacuum gets considerably polarized in the presence of valence quarks.

In order to demonstrate the large spatial extension of the density profiles more clearly and to discuss finite-size effects in the $B=1$ sector we have solved the mean-field equations for different values of the basis parameter $R_{c}$. For this purpose the constituent-quark mass was chosen as $m=600 \mathrm{MeV}$. In Fig. the corresponding baryon density is shown as a function of $r / R_{c}$. After multiplication with a scale factor $R_{c}^{3}$ the profiles look very similar, thus indicating the basis dependence and spatial extension of the solutions. In terms of the total energy of the configurations, however, a radius of $R_{c}=8 \mathrm{fm}$ seems 


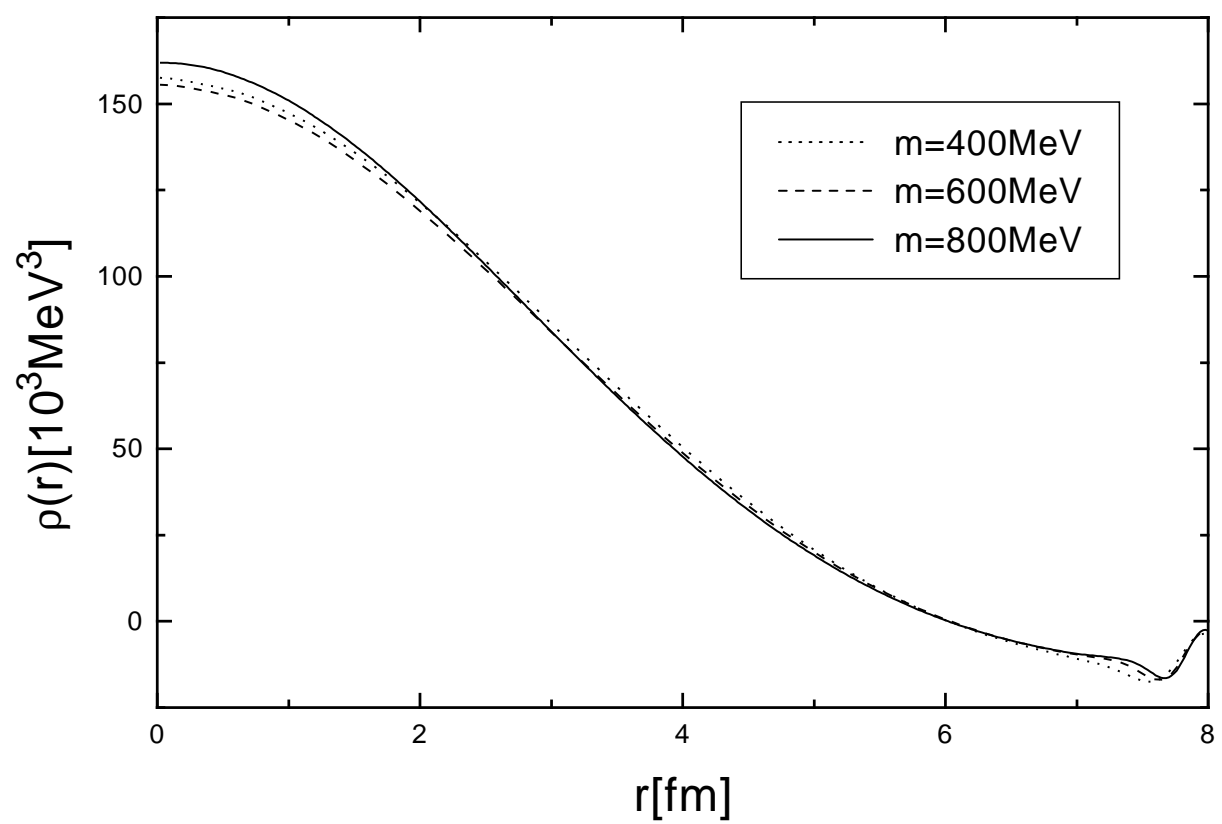

Figure 3: $3\left\langle\Psi^{\dagger} \Psi\right\rangle$ for different constituent-quark masses

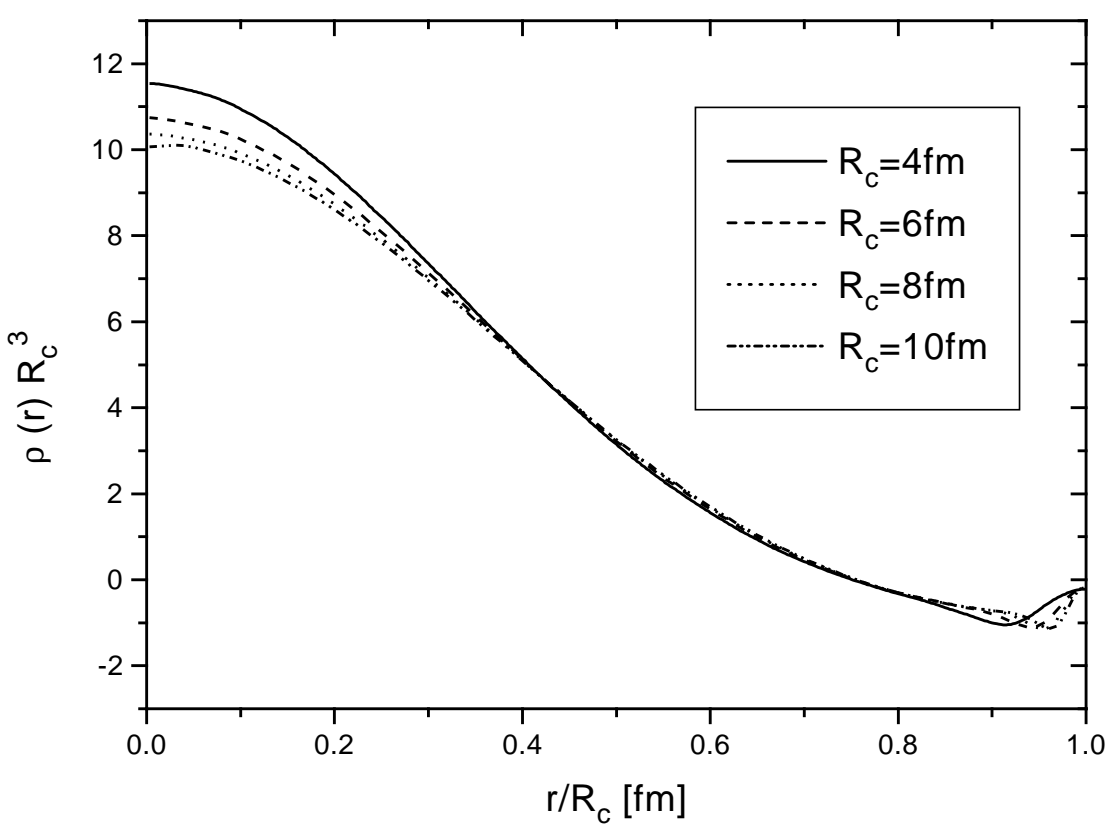

Figure 4: $\quad 3\left\langle\Psi^{\dagger} \Psi\right\rangle R_{c}^{3}$ for different values of $R_{c}(m=600 \mathrm{MeV})$. The factor $R_{c}^{3}$ accounts for the normalization condition and was included in order to compare the different profiles. 


\begin{tabular}{|c|c|c|c|c|}
\hline$R_{c}[\mathrm{fm}]$ & 4 & 6 & 8 & 10 \\
\hline$E_{\text {tot }}[\mathrm{MeV}]$ & 4454 & 4542 & 4586 & 4587 \\
\hline
\end{tabular}

Table 1: The total energy of the $B=1$ configuration as a function of the basis parameter $R_{c}(m=600 \mathrm{MeV})$.

sufficient to reproduce the continuum limit with reasonable accuracy. This is indicated by the functional dependence of the energy on $R_{c}$ which is displayed in Table 1 .

It is helpful to map the degrees of freedom in our approach to those used in the NJL soliton model. The simplest version of the latter uses a scalar-isoscalar field $\sigma$ and a pseudoscalar-isovector field $\boldsymbol{\pi}$. In terms of mode sums as in Eqs. 8 and 9 these fields are given by

$$
\begin{aligned}
\sigma & =\sum_{i} \bar{\Psi}_{i} \Psi_{i} R^{2}\left[\epsilon_{i}\right] \bar{\rho}(i) \\
\boldsymbol{\pi} & =\sum_{i} \bar{\Psi}_{i} \gamma_{5} \tau \Psi_{i} R^{2}\left[\epsilon_{i}\right] \bar{\rho}(i) .
\end{aligned}
$$

By construction of $\bar{\rho}$, we obtain $\boldsymbol{\pi}=0$. In the NJL soliton model the fields are usually constrained to the chiral circle $\sigma^{2}(\mathbf{r})+\boldsymbol{\pi}^{2}(\mathbf{r})=f_{\pi}^{2}$, where $f_{\pi}$ is the pion decay constant. When this constraint is dropped the solitonic solutions have been found to become unstable and the $\sigma$ field develops a sharp structure localized around the origin [22, 23]. However, it was then shown in [24] that the collapse can be prevented by properly fixing the baryon number.

Clearly such results are relevant in the present context, since by varying the $\sigma$ field and constraining the pion field to zero we leave the chiral circle. In all the calculations performed we have never observed a collapse of the field configuration. Even when the baryon-number constraint is dropped a localized field configuration does not develop. Note, however, that the prescription for fixing the baryon number is different from the one used in Ref. [24].

Based on the results obtained by Sieber et al. [22] a simple explanation for this observation can be given. They have found that the collapse of the soliton is related to the valence level being lowered to negative energies, whereas the rest of the spectrum remains basically unchanged. A necessary condition for such a phenomenon to occur is the violation of charge conjugation invariance. In [22] charge conjugation invariance is broken due to a nonvanishing pion field. However, the mean-field Hamiltonian we use does not contain pionic degrees of freedom. In addition to that, the model space we have defined in order to numerically solve the Dirac-Hartree-Fock problem is $C$-invariant. As a result, the symmetry of the single-particle spectrum under inversion of the energy axis is reproduced with high accuracy and a collapse of the fields driven by a level plunging into the Dirac sea can never occur.

From the results for the $B=1$ sector we conclude that the pseudoscalar contribution to the self-energy is of major importance for the emergence of the soliton in the grand- 
spin approach. In our model the pseudoscalar field is constrained to vanish by reasons of symmetry. In the NJL soliton model the self-consistent solutions exhibit a sizeable, surface-peaked pion field [25], which in a bosonized formulation takes the role of the pseudoscalar mean field.

In order to quantify the importance of pionic contributions we have estimated the relative size of the different terms of Eq. (3) in the ground state of the nucleon. The pionic contributions vanish by averaging the density matrix of the system, so the idea is to evaluate the corresponding matrix elements before taking the average. We thus consider a state vector with three valence quarks coupled to $\left(J^{p}, T\right)=\left(\frac{1}{2}^{+}, \frac{1}{2}\right)$, as discussed in part I of the paper. The valence wave function is taken from a typical soliton configuration in the NJL model, where only the $\sigma$ field is taken into account. The latter restriction is necessary in order to extract single-particle states with the desired symmetry properties. By means of such a construction we try to mimic a hypothetic solitonic solution which is consistent with the specific assumptions our work is based on.

More specifically, we use a Woods-Saxon parametrization of the selfconsistent field obtained by Meissner et al. 25] with the chiral constraint and a constituent-quark mass of $m=600 \mathrm{MeV}$. Such a field configuration should be a fairly good representative for soliton solutions which are dominated by the valence quarks. For simplicity we assume the absence of vacuum polarization and, in the spirit of an estimate, determine the properties of the system by the valence configuration alone.

The interaction defined in Eq. (3) picks up contributions of different Lorentz and isospin structure. In order to estimate the importance of the different terms for the binding of the soliton we have computed the expectation value of the corresponding contribution to the interaction Hamiltonian. The coupling constants $G_{s}$ and $G_{v}$ can in general be regarded as independent parameters.

As an important result we find that the net attraction is provided by the scalar-pseudoscalar channel, whereas the sum of the vector-axialvector terms gives a positive contribution to the total energy. The scalar-isoscalar and pseudoscalar-isovector terms are both attractive and for the chosen ground-state configuration we find

$$
\frac{\left\langle N\left|\int d^{3} r\left(\bar{\Psi} \gamma_{5} \boldsymbol{\tau} \Psi\right)^{2}\right| N\right\rangle}{-\left\langle N\left|\int d^{3} r(\bar{\Psi} \Psi)^{2}\right| N\right\rangle} \approx 2.4 .
$$

The pionic term is thus expected to add a contribution to the binding of a hypothetic soliton at least as important as the scalar-isoscalar term. When the constituent-quark mass and thus the depth of the potential well is varied between 400 and $800 \mathrm{MeV}$ the ratio of the different contributions stay in the same order of magnitude and the above statements on the sign of the terms remain valid.

It can thus be concluded that our results for the $B=1$ sector are in accordance with such a simple estimate on the binding effects due to a local, chiral interaction. Our numerical investigations show that neglecting the attractive pionic component leads to a drastic change in the structure of the ground state. In view of a realistic description of the nucleon within the NJL model the hedgehog ansatz, allowing for a nonvanishing pion field, is clearly superior to our treatment of symmetries. 


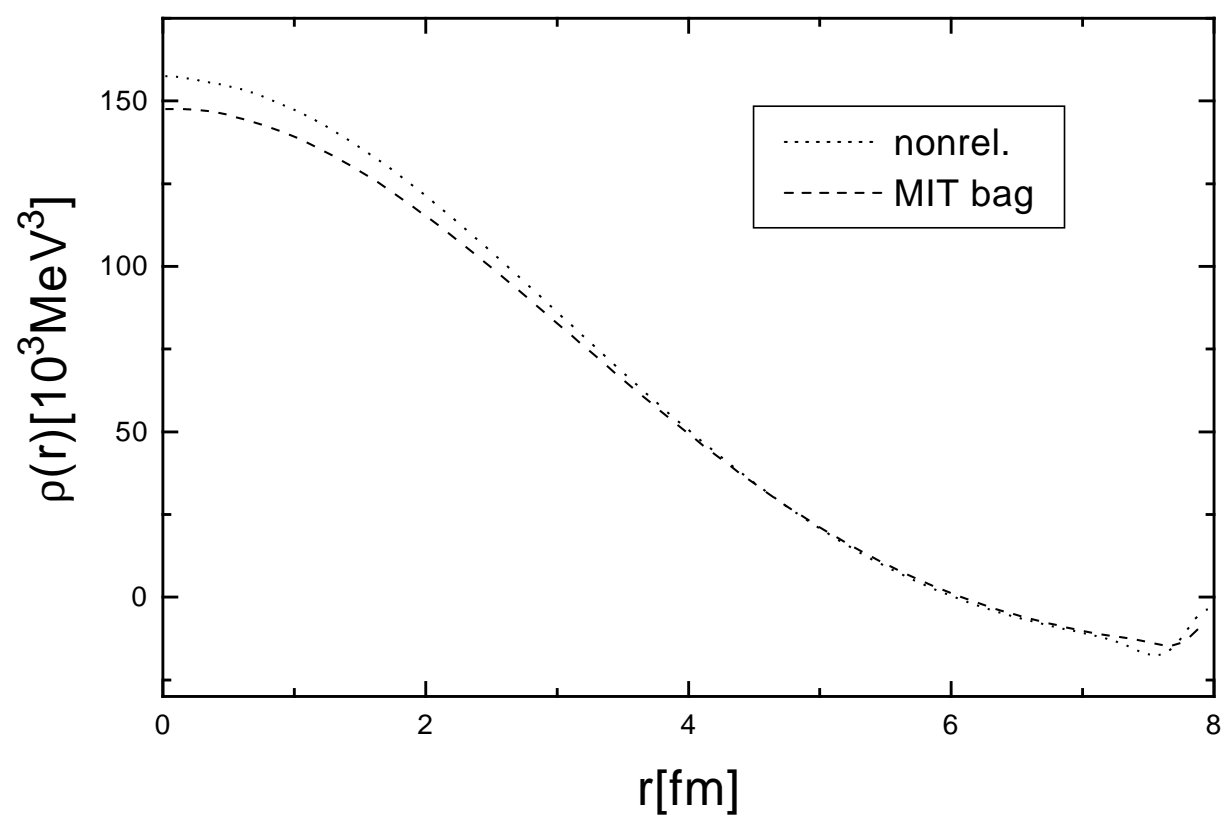

Figure 5: $3\left\langle\Psi^{\dagger} \Psi\right\rangle$ as obtained in model spaces with different boundary conditions. The constituent-quark mass was fixed to $m=400 \mathrm{MeV}$.

However, a few aspects of the approach have not yet been illuminated. It has been pointed out that topologically nontrivial solitonic field configurations should be represented in a model space of states that satisfy appropriate boundary conditions [21]. The basis space suited for, e. g., scalar and pseudoscalar fields is different from the appropriate space for vector and axial-vector fields. Topological features of our field configurations cannot be discussed in analogy to the NJL soliton model, where topologically distinct classes of fields are characterized by different winding numbers of the chiral angle. Since the pion field is missing, we do not have the same classification of topological structures as in the NJL soliton model. Still we want to examine the influence of the boundary condition imposed on the basis states on the structure of the self-consistent solutions. In Fig. 5 the baryon density as obtained with the nonrelativistic and the MIT-bag boundary condition is displayed. The two density profiles are almost identical. The energy obtained with the MIT-bag boundary condition is slightly higher $(3855 \mathrm{MeV}$ vs. $3769 \mathrm{MeV})$. As already found in the vacuum sector, the boundary condition only has a minor influence on the structure of the solutions.

When we switch on the vector potential, the density profile becomes broader (Fig. 6). This is expected from the repulsive character of the vector interaction in the particleparticle channel. The energy of the configurations is increased compared to the value obtained with $G_{v}=0$. When $G_{v}$ exceeds a critical value $G_{v}^{c r i t} \approx G_{s}$ the iteration scheme fails to converge. An interesting situation occurs when the sign of the vector interaction is reversed. Clearly such a situation is unphysical, since the vacuum becomes unstable for a strongly repulsive $q \bar{q}$ interaction. However, before the instability sets in a localized, solitonic system is formed. While lacking any physical relevance the formation of a bound state can be viewed as a test for the numerical implementation of the Dirac-Hartree-Fock 


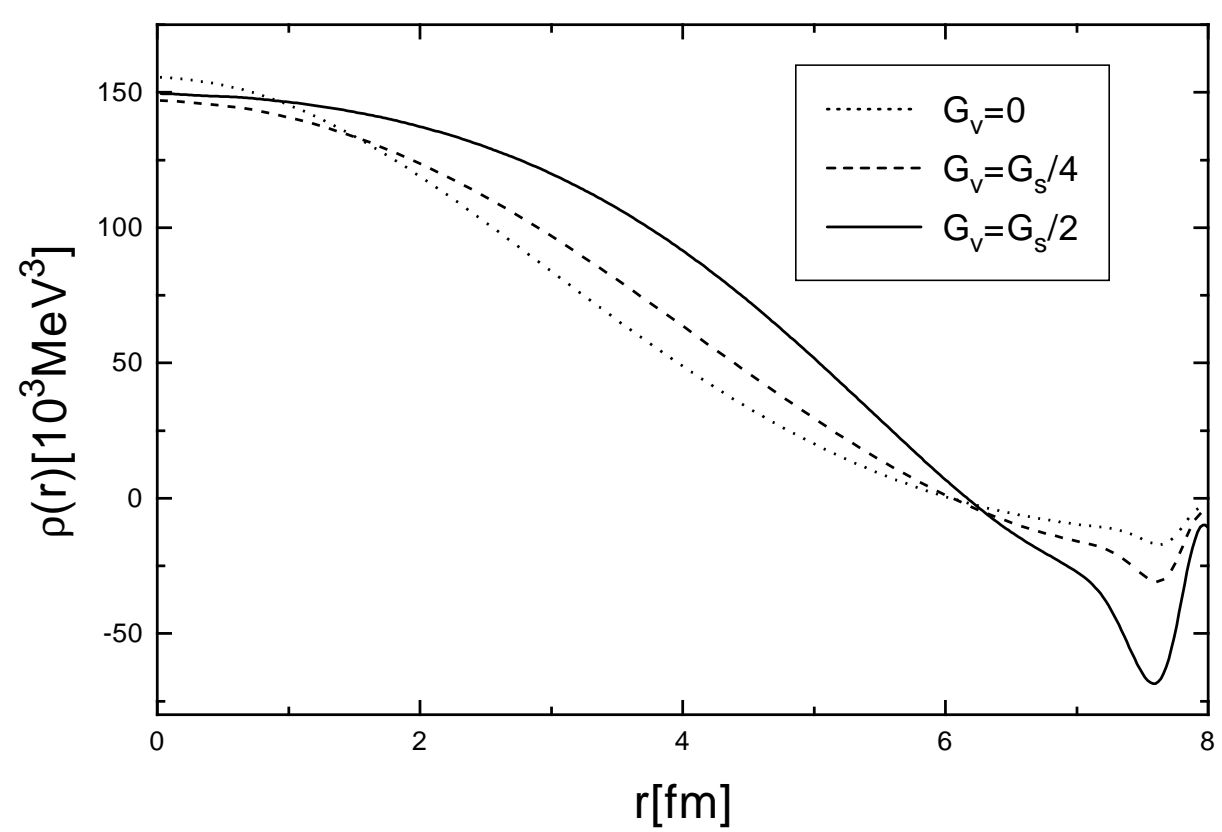

Figure 6: $3\left\langle\Psi^{\dagger} \Psi\right\rangle$ for different values of $G_{v}(m=600 \mathrm{MeV})$

approach. It thus becomes evident that the basis space contains the necessary highfrequency components to resolve a well-localized object.

With no other degrees of freedom besides the scalar and the vector channel accessible, we conclude that the NJL model does not possess solitonic solutions in the $B=1$ sector if a rotationally and isospin invariant mean-field configuration is used. It is crucial for the emergence of the soliton to truncate the symmetry group to an invariance under simultaneous rotations in isospin and coordinate space, as inherent in a grand spin zero configuration.

The results indicate that the prescription to obtain an invariant ground-state density by means of spin-flavor averaged density matrices is insufficient in the context of the NJL model. This conclusion, however, might loose its validity within a more general class of models. The phenomenological success of the MIT-bag model as well as the nonrelativistic quark model [26] indicates that confining forces play a crucial role for the binding of baryons. The confining forces were completely neglected so far, because the NJL model allows free quarks as asymptotic states. A major goal for the further development of chiral quark models is therefore the incorporation of a self-consistent description for confinement.

\subsection{Modeling Confinement}

The ground-state configurations obtained for the $B=1$ system are not suited for the phenomenological investigation of the nucleon, since on mean-field level the valence quarks are not bound.

Our main interest is the excitation spectrum of nucleon rather than the ground state. In 
an RPA calculation the information about ground-state structure enters via the singleparticle energies and wavefunctions. We therefore need to construct a model for the nucleon from which we extract the necessary information.

The situation is similar as in the early days of RPA calculations for low-lying excitations of nuclei. There a phenomenological single-particle basis was used together with a parametrization for the residual interaction [27, 28]. Despite the lack of consistency excitation energies and transition strengths could be reproduced quite successfully with such an approach.

As a prototype of a simple model for the nucleon we consider the MIT-bag model [29], where the determination of a set of positive- and negative-energy eigenstates reduces to the task of solving a nonlinear equation. However, for the examination of the dynamical response of the nucleon this model seems to be too crude. According to the tensor-RPA description the RPA-excited states might have contributions from monopole modes admixed whenever this is allowed by angular-momentum selection rules. The monopole modes are described by $p h$ pairs coupled to $J^{P}=0^{+}$and can be viewed as radial oscillations of the ground-state density. We expect that in the MIT-bag model such modes cannot be adequately described due to the rigid bag boundary. The results to be discussed in the next section support the idea that an MIT-bag potential does not provide a suitable ground state for RPA calculations.

In order to account for surface vibrations and monopole modes the MIT bag has to be replaced by a smoother potential. Let us consider scalar potentials of the form

$$
m(r)=c r^{\alpha}
$$

where the MIT bag is recovered in the limit $\alpha \rightarrow \infty$. The excitation spectrum of the system without any ph interaction can be obtained from the single-particle spectrum of the Dirac Hamiltonian containing the scalar self-energy $m(r)$. These states will get shifted when the RPA residual interaction is turned on. It is well known that due to ground-state correlations one or even a couple of these levels will get rapidly lowered (see, e. g., [30]). Hence we expect an enhanced level density at low energies. A linear potential $(\alpha=1)$ will lead to a high density of excited states already on mean-field level and seems problematic for that reason. We therefore decided to choose an harmonic-oscillator potential $(\alpha=2)$. Anticipating some of the results of the next chapter, for a standard nucleon configuration with a root-mean-square radius (rms) of about $0.8 \mathrm{fm}$ the problem of an overabundance of low-lying states cannot be solved by simply picking a specific $\alpha$. In order to obtain a fair description of the baryon spectrum we are forced to consider a quark core of drastically reduced size. It is generally assumed that the experimental value for the root-mean-square radius contains sizeable contributions from the $q \bar{q}$ polarization cloud of the nucleon, the quark core being considerably smaller. Obtaining reliable experimental data for the size of the quark core is extremely difficult. However, a few attempts in that direction have been made, with estimates ranging from $0.2 \mathrm{fm}$ [31] to $0.5-0.6 \mathrm{fm}$ [32].

In our approach the quark core is modeled by the mean-field configuration, whereas the $q \bar{q}$ polarization cloud is accounted for by RPA ground-state correlations. Thus, besides a standard MIT-bag configuration, we consider an oscillator potential giving a nucleon 


\begin{tabular}{|c|c|c|c|}
\hline Potential & $\alpha$ & $\left\langle r_{E}^{2}\right\rangle_{p}^{\frac{1}{2}}[\mathrm{fm}]$ & $E_{\text {val }}[\mathrm{MeV}]$ \\
\hline \hline MIT-bag & $\infty$ & 0.71 & 405 \\
\hline Oscillator & 2 & 0.33 & 1052 \\
\hline
\end{tabular}

Table 2: Root-mean-square charge radius and valence-quark energy for the different parametrizations of the potential

rms of about $0.3 \mathrm{fm}$. In table 2 we display the characteristics of the different models for the ground state. The calculations presented in the next chapter are based on these two alternative descriptions.

For the harmonic-oscillator potential the Dirac equation for the central-force problem was solved numerically by a Runge-Kutta method with adaptive stepsize control. In case of the MIT-bag potential only one nonlinear equation for the eigenvalues had to be solved. In both cases the current-quark mass was fixed to $m_{0}=5 \mathrm{MeV}$. The numerical method for the first order differential equation has been checked by comparison with the results for an MIT-bag potential and a scalar potential of the form $m(r)=c r$ [33 which both can be obtained semi-analytically.

In the simple potential picture there is nothing that makes a valence-quark configuration with the quantum numbers of the nucleon energetically preferable to a $\Delta(1232)$ configuration. For this reason the ground-state energy should be considered as the $N-\Delta$ average. Due to the uncertainty principle we obtain a quite large value for the valence energy in case of the harmonic-oscillator potential. This might be an indication that the total energy of the ground state which picks up a contribution from the single-particle energies is overestimated in this model. Note, however, that in a RPA description the energy is lowered by ground-state correlations [34]. Such correlations account for the deviation of the physical state vector from a simple, antisymmetrized many-body wave function (e. g. a Slater determinant) and always decrease the energy of the ground state. The concept is meaningful even in the context of bag models which cannot be derived as self-consistent solutions of a field theory. In that regard especially the problem of restoration of broken symmetries has been studied. It has been found that, e. g., center-of-mass corrections lead to a significant decrease of the energy of bag states [35]. In the NJL soliton model ground-state correlations have been found to lower the energy of the mean-field configuration by a couple of hundred $\mathrm{MeV}$ 4. However, the RPA is only expected to work well when ground-state correlations are not too sizeable. Otherwise an extended scheme as in Ref. [30] has to be taken into account which incorporates dynamical correlations also in an explicit fashion. This issue will be discussed in more depth in the next chapter.

An effective way to model ground-state correlations would be to consider a small-size MIT-bag configuration with modified boundary conditions on the bag surface, the so called "Little Bag" 36]. The modified boundary conditions are due to the chiral fields outside the bag which, in a complete RPA treatment, are described by $q \bar{q}$ admixtures to the ground state. It can be shown that the chiral fields always lower the energy of the 
valence level [37], thus supporting the arguments given above.

In a much simpler fashion we could shift the single-particle levels without even changing the wavefunctions by introducing a (constant) time-like component of a vector potential. This shows that within the scope of the simple models considered here the valence energy is not a particularly meaningful quantity. It is rather the single-particle excitation energies which are relevant in an RPA context. The latter are clearly insensitive to a shift of the energy scale.

Our choice of a central mean-field potential is compatible with the symmetries we demand for the eigenstates. Besides the restrictions for rotational and isospin symmetry set by the tensor-RPA method we require parity eigenstates. These requirements can as well be fulfilled by a potential transforming as the time-like component of a Lorentz vector. However, the inclusion of a vector potential in the Dirac equation might lead to solutions which are not normalizable. Recently it was shown that the wavefunctions obtained in a linear vector potential acquire a non-integrable singularity, whereas the solutions in the corresponding scalar potential are well behaved [38. In order to avoid such problems and to keep the model for the ground-state as simple as possible we restrict ourselves to scalar potentials. The eigenenergies and eigenfunctions obtained with the above parametrizations serve as an input for the tensor-RPA method. In the next section we discuss a first application of this method to the excitation spectrum of the nucleon.

\section{The Excited States}

Although in the last 20 years a variety of models for baryons have been proposed [39], we are still far from a complete understanding of the baryon spectrum. Experimentally about 20 resonances below $2 \mathrm{GeV}$ are confirmed in the non-strange sector.

The phenomenologically most successful models have their origin in the nonrelativistic quark model [26] which was systematically applied to the problem of baryon spectroscopy by Isgur and Karl [8]. Initially, such formulations contained three constituent quarks which interact via a harmonic-oscillator potential and a residual interaction of spin-color type which is treated perturbatively. With such an ansatz the baryon spectrum in the negative-parity channel could be satisfactorily reproduced, whereas with the same parameter set no acceptable description of the positive-parity data was obtained.

The "parity problem" was overcome only recently when the formulation of the constituentquark model was modified. On the level of perturbation theory a satisfactory description of the data in both parity channels is obtained when the spin-color interaction of Isgur and Karl is replaced by a spin-flavor interaction [15]. A nonperturbative treatment of a generalized two-body interaction in a model space leads to a good overall agreement with the data as well [40]. Despite these successes the constituent-quark models leave a number of questions unresolved.

First of all it still remains difficult to establish a link to QCD, since the elementary degrees of freedom are constituent quarks which are quite complicated objects in terms of the current quarks of QCD [41].

Besides this conceptual problem there are two issues to be clarified. As a result of most 
formulations of the constituent-quark model unobserved states occur in the spectrum. It needs to be clarified if these states exist in nature and have not yet been detected experimentally or if they are incorrectly predicted by the constituent-quark model. The problem of unobserved states seems to be quite general and is also encountered in attempts to describe the baryon spectrum in the MIT-bag model even for relatively small model spaces [42, 43].

The other problem that needs further inspection is related to vacuum structure. The mass scale of baryon resonances predicted by the constituent-quark model is such that sizeable admixtures of $q \bar{q}$ states are expected even for quark masses of the order of $200-300 \mathrm{MeV}$. Such contributions are completely neglected in the constituent-quark model. The assumption that the spectral functions of QCD are dominated by the lowest excitations of the QCD vacuum leads to a low-energy effective theory known as chiral perturbation theory 44]. The phenomenological success of chiral perturbation theory indicates that such an assumption seems well justified 45. Thus mesonic admixtures to the baryon wavefunctions are easily conceivable. The Skyrme model [46] constitutes an extreme but quite successful description of baryons in terms of meson fields only.

The tensor-RPA method as developed in part I of this paper allows to treat the valence excitations and the excitations of the Dirac sea in the same framework. It may serve as a bridge from constituent-quark models to chiral models.

The tensor-RPA matrix elements split up into a mean-field and a residual interaction part. The mean-field part is defined by single-particle energies $\epsilon_{k}$ which we take from the solution of the Dirac equation for the confining potentials discussed in the previous section.

For the residual interaction part we need to specify a particle-hole $(p h)$ interaction. A simple choice that has proven to be applicable in the context of a RPA description of mesons [7] is the color-current interaction Eq. (11). As a second model we use an NJLtype interaction in the $\sigma$ - and $\boldsymbol{\pi}$-channel

$$
\mathcal{L}_{\text {int }}=G\left[(\bar{\Psi} \Psi)^{2}-\left(\bar{\Psi} \gamma_{5} \boldsymbol{\tau} \Psi\right)^{2}\right] .
$$

The Lagrangian defined above is invariant under $S U(2)_{L} \times S U(2)_{R}$ chiral transformations. Recently it has been claimed by Glozman and Riska that a pseudoscalar meson exchange between constituent quarks could explain the structure of the baryon spectrum [15]. With the interaction Eq. (27) we have effectively introduced a local pion and sigma exchange between current quarks. Such a Lagrangian can be extracted from the Sigma Model when the meson masses are going to infinity [39]. The arguments in favor of a pseudoscalar meson exchange interaction given in Ref. [15] were based on the group-theoretical structure of the nonrelativistic reduction of their model rather than on the detailed form of such an interaction. Our goal is to study the effects of pseudoscalar meson exchange on current-quark level with the above Lagrangian.

A chirally invariant point-like interaction between quarks has been motivated from various approaches describing the QCD vacuum [47, 48]. All these approaches neglect the backreaction of the gluon fields to quark sources and only describe quark interactions in the background of the nonperturbative gluon vacuum. In the extreme case of an MIT- 
bag ground state the quarks expel the gluon condensate from a spherical cavity leaving the perturbative vacuum inside. In such a scenario the use of a perturbative one-gluon exchange interaction seems more natural than a NJL interaction. A finite-range interaction would lead to retardation effects which are not accounted for in the present RPA formulation. A simple estimate shows that for the range of excitation energies we are interested in retardation effects might play an important role. Therefore we consider a local color-current interaction as an equally suitable description as a finite-range gluon exchange without retardation.

The combination of one of the different mean-field configurations discussed in the previous section with a $p h$ interaction of local color current or local $\sigma-\boldsymbol{\pi}$ type clearly does not constitute a consistent, symmetry conserving Dirac-RPA scheme. Hence we expect spurious admixtures in the excitation spectrum. However, such admixtures can be excluded by restricting the calculation to excited states with positive parity. In the small amplitude limit a state generated by acting with a symmetry transformation on a positive-parity ground state carries the same parity quantum numbers as the generators of the symmetry group

$$
\begin{aligned}
\exp \left(i \theta_{a} S_{a}\right)|N\rangle & =|N\rangle+|\Delta N\rangle \\
P|\Delta N\rangle & \approx i \theta_{a} P S_{a} P|N\rangle .
\end{aligned}
$$

In these expressions $S_{a}$ denotes the generators of the Lie-group under consideration and $P$ is the parity operator. In our model for the nucleon the relevant symmetries which are broken in the ground state are translational and chiral invariancet. Chiral symmetry is broken by the scalar mean-field potential. The generators of these groups are both negative-parity operators, so that in the positive-parity channels the RPA states are orthogonal to the spurious modes.

Since the ground state is modelled by solutions of the Dirac equation we are in a position to account for $q \bar{q}$ components in the wavefunctions of the excited states. Note, however, that the Dirac sea in such a description is given by localized negative-energy states. Thus our ansatz includes those mesonic excitations that are strongly correlated with the quark core of the nucleon. It would be desirable to find a RPA description for the whole spectrum of mesonic excitations including the pseudoscalar Goldstone modes of the QCD vacuum which are decoupled from the regions of the Dirac sea polarized by the quark core. However, such a general approach within a confining quark model seems extremely difficult and is left for future investigations.

For the numerical realization of the tensor-RPA scheme we compute the RPA matrix $M$ and the metric tensor $N$ as defined in part I for various combinations of the mean-field potentials and the $p h$ interactions discussed above. In a compact notation, the RPAequation is given by [1]

$$
\left(\begin{array}{cc}
M^{(1)} & -M^{(2)} \\
-M^{(3)} & M^{(4)}
\end{array}\right)\left(\begin{array}{c}
X \\
Y
\end{array}\right)=\omega_{\Delta}\left(\begin{array}{cc}
N^{(1)} & -N^{(2)} \\
-N^{(3)} & N^{(4)}
\end{array}\right)\left(\begin{array}{c}
X \\
Y
\end{array}\right) .
$$

\footnotetext{
${ }^{2}$ Due to the small current-quark masses chiral symmetry is also broken explicitly. Our discussion applies to the case of vanishing current-quark masses
} 
with

$$
\begin{aligned}
M_{(m i) k,(n j) l}^{(1)} & =\sum_{\Gamma} C_{k, l}^{\Gamma}\left\langle\Gamma_{N}\left\|\left[A_{m i}\left(\bar{\Gamma}_{k}\right), H, A_{n j}^{\dagger}\left(\Gamma_{l}\right)\right]^{\Gamma}\right\| \Gamma_{N}\right\rangle \\
M_{(m i) k,(n j) l}^{(2)} & =\sum_{\Gamma} C_{k, l}^{\Gamma}\left\langle\Gamma_{N}\left\|\left[A_{m i}\left(\bar{\Gamma}_{k}\right), H, A_{n j}\left(\bar{\Gamma}_{l}\right)\right]^{\Gamma}\right\| \Gamma_{N}\right\rangle \\
M_{(m i) k,(n j) l}^{(3)} & =\sum_{\Gamma} C_{k, l}^{\Gamma}\left\langle\Gamma_{N}\left\|\left[A_{m i}^{\dagger}\left(\Gamma_{k}\right), H, A_{n j}^{\dagger}\left(\Gamma_{l}\right)\right]^{\Gamma}\right\| \Gamma_{N}\right\rangle \\
M_{(m i) k,(n j) l}^{(4)} & =\sum_{\Gamma} C_{k, l}^{\Gamma}\left\langle\Gamma_{N}\left\|\left[A_{m i}^{\dagger}\left(\Gamma_{k}\right), H, A_{n j}\left(\bar{\Gamma}_{l}\right)\right]^{\Gamma}\right\| \Gamma_{N}\right\rangle,
\end{aligned}
$$

where $H$ is the full Hamlitonian and $C_{k, l}^{\Gamma}$ are recoupling coefficients. The metric tensor $N$ is obtained from eq. (30) when the Hamiltonian is omitted and the double commutators are replaced by ordinary commutators. For the computations presented here we use the exact expressions for the many-body matrix elements of Eq. (30) as given in part I.

In order to check the rules for the reduction of the overcomplete $p h$ space we have computed the metric tensor in the full space of $p h$ states. It was found that whenever a set of energetically degenerate states was linearly dependent according to the reduction scheme the metric tensor $N$ was singular in that subspace. When the full space of dimension $d$ was reduced to the smaller dimension $d_{r}$, the determinant taken in the reduced space became nonzero.

The results for the excitation spectrum presented here were obtained with a $p h$ basis of dimension 50. With a larger model space the energies of the lowest excitations are shifted. However, this shift can be compensated by rescaling the coupling constant. At the rescaled value of $G$ the spectrum looks very similar to that which was obtained with the standard basis of dimension 50. This observation seems to indicate that the dynamics of the system depends only on a certain combination of the coupling constant and the cut-off in ph space. A similar behaviour is found in the vacuum sector of the NJL-model, where the relevant parameter is $G \Lambda^{2}$ [16]. Note, however, that in the latter case $\Lambda$ defines a cut-off in the single-particle energy.

For any excited state with fixed quantum numbers $\left(J^{p}, T\right)$ the model space is spanned by the $p h$ states with lowest energy with respect to the mean-field potential. Valence excitations and excitations of the Dirac sea contribute in an equal manner. The $p h$ states are chosen according to angular momentum/isospin/parity selection rules, including any $p h$ combination which can be coupled with the ground state to the desired quantum numbers of the excited state. Together with the reduction scheme for linearly dependent states this guarantees the completeness of the $p h$ space when the basis dimension goes to infinity.

In Fig. 7 the excitation spectrum for the MIT-bag mean field obtained with different $p h$ interactions is displayed. The excitation energies for various $(J, T)$ channels are plotted as a function of the coupling constant $G$. Note that the $\left(\frac{3}{2}^{+}, \frac{3}{2}\right)$ channel was not considered due to the specific problems discussed in part I.

As a global feature we observe that the states are lowered in energy by the $p h$ interaction. In most cases the decrease of the first excited state to zero energy occurs within a narrow range of coupling constants. Eventually the level meets its negative-energy counterpart 

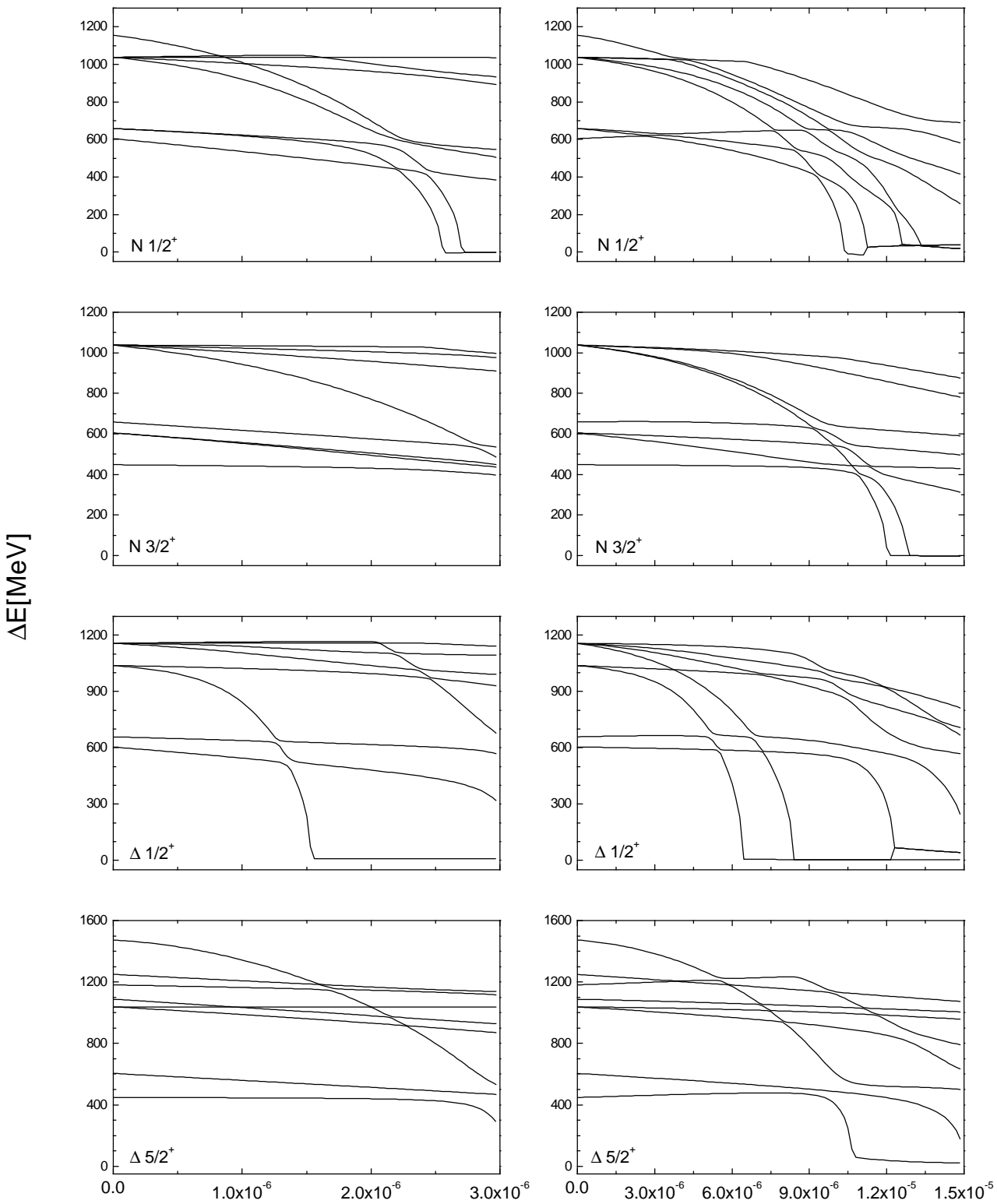

$\mathrm{G}\left[\mathrm{MeV}^{-2}\right]$

Figure 7: The excitation energies as a function of $G$ for the MIT-bag mean field. Left column: $\sigma-\pi$ interaction. Right column: Color-current interaction. 
(not shown) which has been raised. The fact that the two levels meet at an energy approximately equal to zero indicates that the spectrum displays a symmetry under inversion of the energy axis. A closer inspection of the whole spectrum including negative-energy states shows that this symmetry is approximately preserved until the first level is lowered to zero. A serious violation of the symmetry has never been found in all the spectra we have computed.

The results for the color-current interaction are quite similar to those for the $\sigma-\boldsymbol{\pi}$ interaction when the coupling constant is rescaled. As a difference the color-current interaction is more repulsive in the channels under investigation and the energy of some states is slightly increased.

Comparing these results to the experimental data we find that for a standard MIT-bag configuration the level density of $p h$ states is too high already on mean-field level $(G=0)$. Whereas the absolute scale for the energies might not be very well defined due to possible shifts of the ground-state energy (which is lowered by correlations), the essential problem lies in the small spacing between various states.

Even when the residual interaction is switched on the spectra never resemble the experimental data for the baryon spectrum. On the contrary, the level density gets further enhanced. An eigenvalue which meets its negative-energy counterpart at a certain value of $G$ develops a finite imaginary part for larger values of the coupling constant. Only the real parts are shown in the figures.

Another global feature of the excitation spectra is the occurrence of quasi-crossings. According to a theorem of Wigner and von Neumann 49 levels belonging to the same representation of a symmetry group cannot cross under the influence of a perturbation. A closer inspection of the excitation spectra obtained with the tensor-RPA method shows that some levels approach up to a distance of a few $M e V$ but do not cross.

In order to obtain information on configuration mixing in the spectrum we have studied the eigenvectors. We only report some general features of the $p h$ amplitudes and avoid displaying the vast amount of data.

When the levels are well separated and ground-state correlations are negligible there is always a dominant contribution of one $p h$ state to the eigenvectors. Configuration mixing becomes sizeable when the levels lie close together.

Ground-state correlations within an RPA approach are characterized by nonvanishing $Y$ amplitudes. We find that for coupling constants up to values close to the point where the first eigenvalue becomes degenerate with the ground state the $Y$-amplitudes are negligible. A characteristic feature of an MIT-bag and other stiff potentials is the fact that the first excited state is lowered to zero energy within a very narrow range of coupling constants. Only within this range an eigenvector develops sizeable $Y$-amplitudes. The largest $Y$-values are found for $p h$ excitations from the Dirac sea, reflecting the strong coupling to negative-energy states due to a relatively small energy gap. Hence the level is driven down by ground-state correlations originating from vacuum polarization. Ground-state correlations have been explicitly computed in a simplified RPA scheme ignoring tensor coupling [50]. It has been found that such correlations are sizeable and contributions from the Dirac sea are important. 
The decrease of the first excited state in the manner of a phase transition is a phenomenon well known in RPA theory. Model calculations [30] indicate that this "phase transition" which is accompanied by an overestimate of ground-state correlations is unphysical. With a refined approximation scheme accounting for a more detailed description of the ground state ("self-consistent RPA") it can be shown that the "phase transition" is replaced by a smoother transition to low energies. The standard RPA breaks down at the phasetransition point beyond which the assumptions it is based on are no longer valid. However, we expect that before that point is reached important aspects of the excitation spectrum as the existence of low lying collective states are correctly reproduced.

As already mentioned, the negative-parity states are expected to contain spurious admixtures. For this reason they are not displayed here. In general the mean-field excitation energy is lower for negative-parity states than for those of positive parity. When the residual interaction is switched on the negative-parity levels are driven to zero excitation energy faster then the positive-parity levels, irrespective of the $p h$ interaction which is used. This fact stands in contradiction to the experimental data, where the lowest excitation in, e. g., the $\left(\frac{1}{2}^{+}, \frac{1}{2}\right)$ channel lies below that of the $\left(\frac{1}{2}^{-}, \frac{1}{2}\right)$ channel.

A similar problem with negative-parity states is encountered in the nonrelativistic quark model [15]. Without perturbation the negative-parity excitations lie well below those with positive parity. The problem can be cured by introducing a nonrelativistic reduction of a meson-exchange interaction. When such an interaction is treated perturbatively the order of positive- and negative-parity states gets reversed. In the relativistic case and allowing for configuration mixing of $p h$ states, no such level inversion is found, as can be deduced from our investigations with the $\sigma-\boldsymbol{\pi}$ interaction.

The question arises which feature of the relativistic RPA theory is responsible for this deviation from the nonrelativistic case. In the nonrelativistic framework the level inversion has a quite solid foundation. Within the constituent-quark model its occurrence can be proven by purely group-theoretical arguments while treating the meson-exchange interaction in lowest-order perturbation theory [15]. When the three-body Faddeev equation is solved for the same model the correct level ordering of positive- and negative-parity states is still reproduced [51.

In order to compare these calculations to our approach it is helpful to disentangle the aspect of many-body approximation schemes from the form of the interaction employed. Since the spin-flavor structure of the interaction is the keypoint of the level-ordering problem, we will concentrate on this aspect exclusively. The $\sigma-\boldsymbol{\pi}$ interaction of Eq. 27 does not reduce to the meson-exchange interaction of Glozman et al. in the nonrelativistic limit. An obvious difference is the $\sigma$-exchange term which is missing in the constituentquark model. In addition to that the so called tensor term arising from the nonrelativistic reduction of a pion-exchange interaction [52] was neglected in Refs. [15, 51].

A suitable local interaction reducing in the non-relativistic limit to the structure used by Glozman et al., however, is given by

$$
\mathcal{L}_{\text {int }}=\frac{G}{m^{* 2}}\left(\nabla_{i}\left(\Psi^{\dagger} \Sigma_{j} \tau_{k} \Psi\right)\right)^{2}
$$


where the constituent-quark mass $m^{*}$ is treated as a parameter and replaces $m_{0}$ of Eq. 1. $\boldsymbol{\Sigma}_{i}$ denotes the spin matrices of Dirac theory and a summation over $i, j, k$ is assumed. The nonrelativistic limit of the spin-spin interaction Eq. 31 is defined via the lowest-order terms of the expansion in $q / m^{*}$, where a momentum-space representation of the interaction is used and $q$ denotes the momentum transfer. In lowest nonvanishing order of such an expansion we obtain a two-body potential between quarks $i$ and $j$ with a spin-flavor structure of the form $\boldsymbol{\sigma}_{i} \cdot \boldsymbol{\sigma}_{j} \boldsymbol{\tau}_{i} \cdot \boldsymbol{\tau}_{j}$. In spin-flavor space we thus recover a $S U(2)$ version of Glozman's meson-exchange interaction.

The expression of Eq. 31 can be used as a ph interaction for the Dirac RPA problem in an equal manner as this was done for different versions of the NJL model before. In order to approach the nonrelativistic limit at least approximately without completely blocking $p h$ admixtures from the Dirac sea the constituent-quark mass was chosen $m^{*}=400 \mathrm{MeV}$. When solving the tensor-RPA equation for the spin-spin interaction in the $\left(\frac{1}{2}^{+}, \frac{1}{2}\right)$ and $\left(\frac{1}{2}^{-}, \frac{1}{2}\right)$ channel the lowest negative-parity state is again found below the corresponding level with positive parity. In these calculations the MIT-bag mean-field potential was used.

The same investigations were repeated in Tamm-Dancoff (TD) approximation. The TD approximation amounts to solving a tensor-RPA problem of half the dimension, keeping only $M^{(1)}$ and $N^{(1)}$ in Eq. 29. Physically, this means that $p h$ correlations in the ground state are neglected. This difference turns out to be crucial for the ordering of positiveand negative-parity states. In TD approximation the first excited state in the $\left(\frac{1}{2}^{+}, \frac{1}{2}\right)$ channel is found below the corresponding state in the $\left(\frac{1}{2}^{-}, \frac{1}{2}\right)$ channel when the coupling constant $G$ is increased above a critical value.

This observation leads us to suggest a simple explanation for the wrong level ordering which is always obtained in the RPA calculations. Even when the interaction allows for a level inversion ground-state correlations rapidly lower the first excited state and make it impossible to further study the dynamical interplay of $p h$ configurations. These groundstate correlations come into the game well before the coupling constant has reached the critical value corresponding to a level inversion in TD approximation. As a result, the energy of the first excited state in both parity channels is rapidly decreasing and no level inversion is observed.

The results so far discussed in this chapter should be viewed as a case study which helps to shed light on the range of applicability of the tensor-RPA approach and and to explain typical features of the excitation spectra. For this purpose a standard MIT-bag meanfield configuration has been chosen. However, the spectra do clearly not match with experimental data and thus cannot be assigned any physical significance. Considering the overabundance of low-lying states we have obtained we could only try to describe the baryon spectrum by introducing a large number of unobserved states.

A more satisfactory approach is certainly to reduce the size of the quark core. In combination with a comparatively stiff mean-field potential this will effect a shift of the excitation spectrum towards higher energies. The size of the quark core which is needed to fix the excitation energies to values in the range of the experimental data is determined by the $T=\frac{3}{2}$ states. On mean-field level the lowest $T=\frac{3}{2}$ excitation appears at the same energy 
as the corresponding $T=\frac{1}{2}$ state. Experimentally, however, the energy of the lowest $T=\frac{3}{2}$ state tends to be higher than the excitation energy in the $T=\frac{1}{2}$ channel for the same $J^{P}$. The $\Delta(1232)$, which is not considered here, forms an exception to this rule. Taking into account that the $p h$ interaction in general effects a decrease of the excitation energies, the mean-field energy gaps in the $T=\frac{3}{2}$ channel have to exceed those of the lowest excited state. This opens up the possibility to simultaneously describe the $T=\frac{1}{2}$ channel. In order to adjust the mean-field spectrum correspondingly it is necessary to shrink the size of the quark core to about $0.3 \mathrm{fm}$.

The RPA spectra obtained with the harmonic-oscillator potential introduced in the previous chapter are shown in Fig. 8. For these investigations the color-current interaction was used. In order to compare these spectra with experimental data the coupling constant has to be fixed. For this purpose the $\left(\frac{1}{2}^{+}, \frac{1}{2}\right)$ channel provides a suitable guideline. In this channel the most rapid "phase transition" is observed, whereas for higher angular momenta the phase-transition point is shifted to larger $G$ (outside of the $x$-range displayed in the figure). Since beyond that point the RPA approximation breaks down it is only meaningful to consider coupling constants up to $\approx 2.4 \cdot 10^{-6} \mathrm{MeV}^{-2}$. On the other hand, before the "phase transition" the lowest $\left(\frac{1}{2}^{+}, \frac{1}{2}\right)$ excitation is too high in energy whereas the mass splitting between the various states is too low. We are thus naturally led to choose a coupling constant of $G=2.35 \cdot 10^{-6} \mathrm{MeV}^{-2}$ which allows us to approximately adjust the $\left(\frac{1}{2}^{+}, \frac{1}{2}\right)$ channel to the experimental data. At the same value of the coupling constant the excitation energies in various other channels are obtained as displayed in Fig. 9 .

The horizontal bars indicate the energies obtained in the RPA calculation. The rectangles extend over the range of uncertainty for the resonance maximum extracted from $\pi N$ scattering data [53]. The doubly hatched areas represent $4 *$ and $3 *$ resonances, whereas the hatched areas indicate the energy of $2 *$ and $1 *$ resonances. Since some of the data for the latter are contradictory, the corresponding range of energies has been extracted from the most recent experiments in case of doubt. In order to obtain absolute numbers for the baryon masses we have added the computed values for the excitation energies to a nucleon mass of $940 \mathrm{MeV}$.

Obviously the baryon spectrum is reproduced fairly well also in the other channels. In the $\left(\frac{5}{2}^{+}, \frac{1}{2}\right)$ and $\left(\frac{7}{2}^{+}, \frac{3}{2}\right)$ channel we find low-lying states which have not been observed in an experiment. The residual interaction lifts the degeneracy of certain states and effects a significant improvement over the mean-field spectra. This statement, while easily verified for the small-size quark core, is also true in comparison with ground-state configurations with a larger rms. At the same value of $G$ the negative-parity excitations are found at too low energies, as expected from spurious admixtures.

With the $\sigma-\boldsymbol{\pi}$ interaction a comparable description of the baryon spectrum could not be obtained. We found that this interaction tends to lower the energy of all the levels in a very similar fashion, thus preserving the structure of the mean-field spectrum. We want to emphasize that for obtaining the spectra we have not yet exhausted the full variational space of the most general pointlike chiral interaction. In Ref. [7] such a variational procedure was undertaken for the meson spectra, with the result that the color-current 

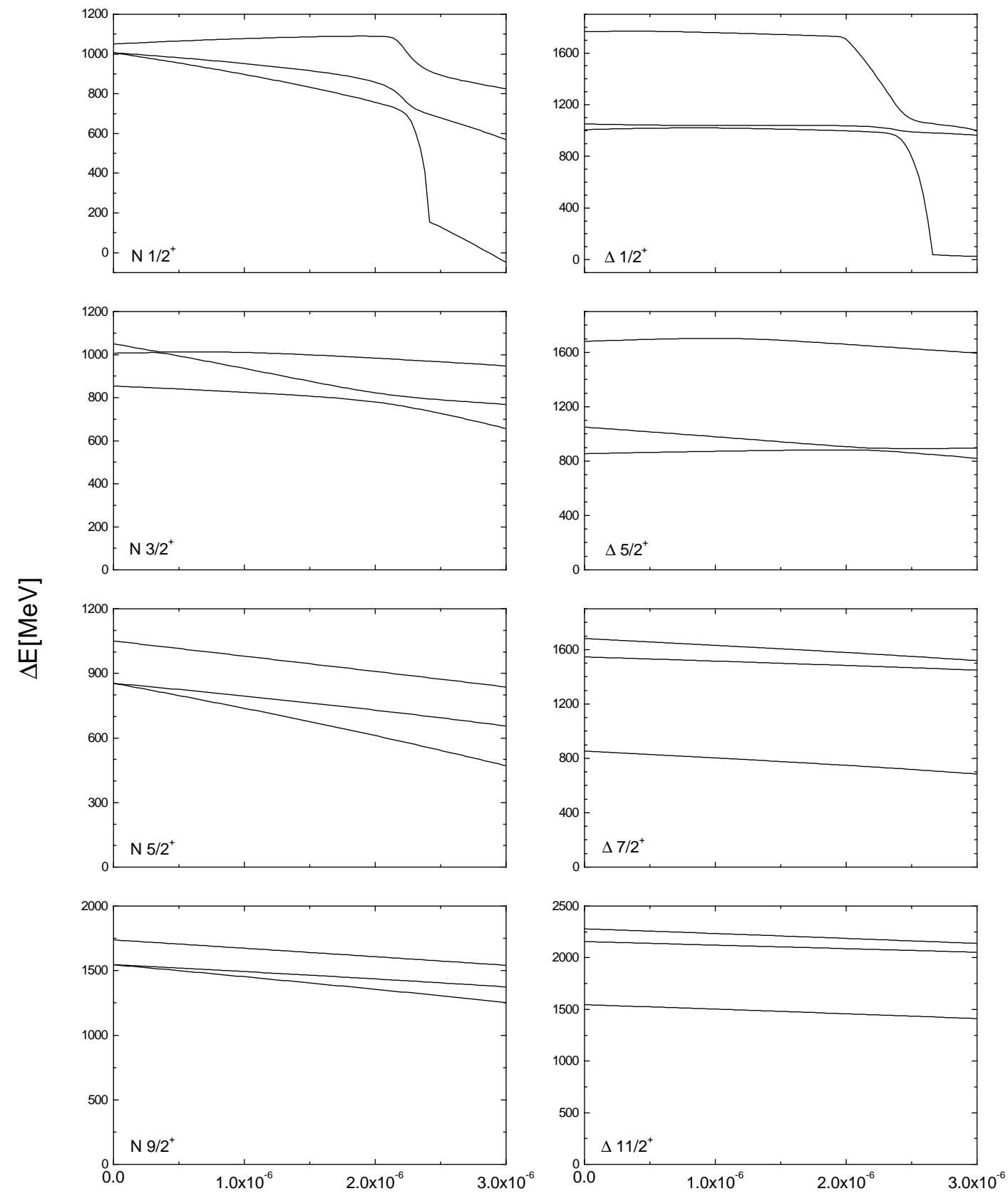

\section{$\mathrm{G}\left[\mathrm{MeV}^{-2}\right]$}

Figure 8: The excitation energies as a function of $G$ for the harmonic-oscillator mean field in combination with the color-current interaction. 


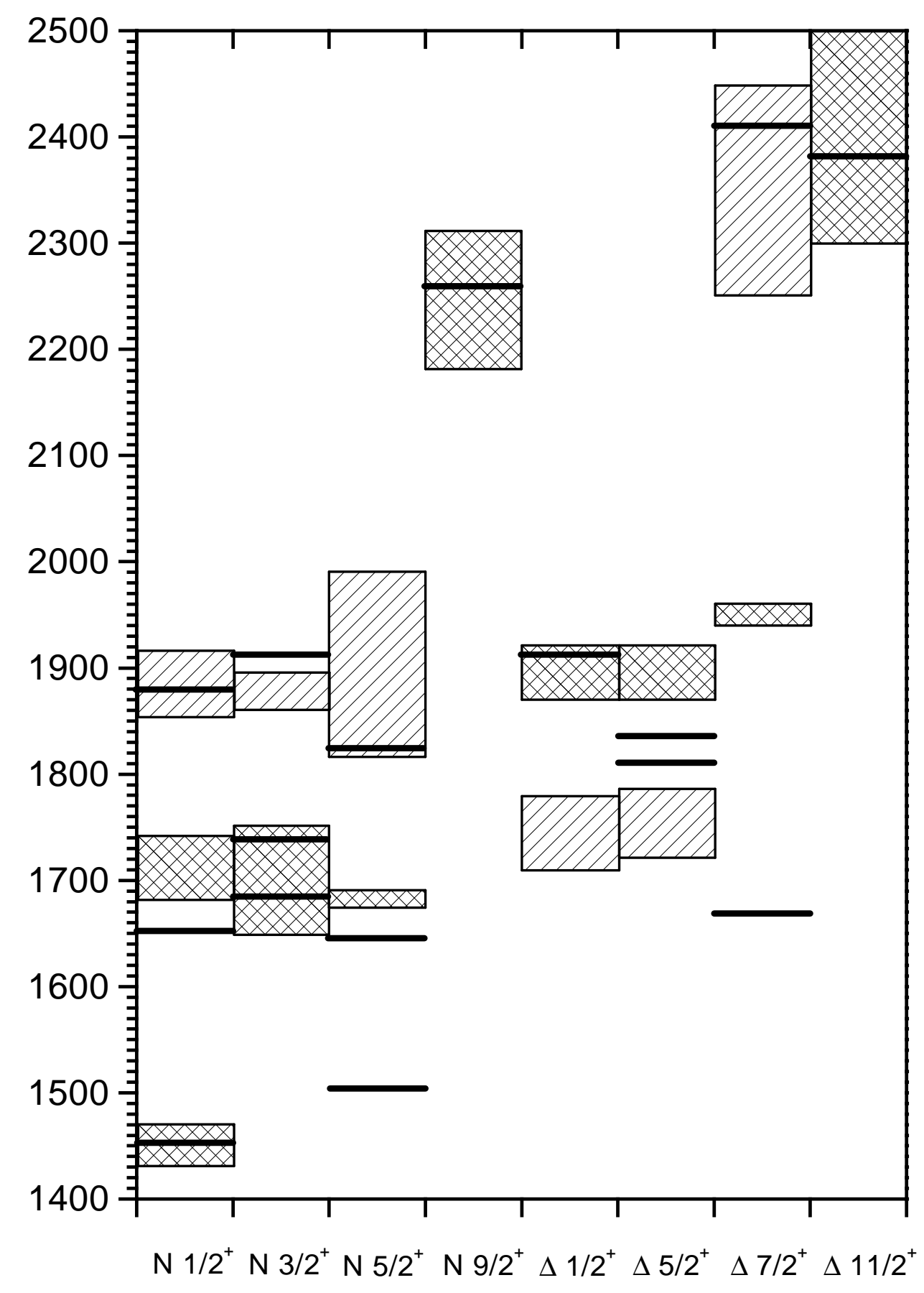

Figure 9: The spectrum of positive-parity baryons obtained with the color-current interaction at $G=2.35 \cdot 10^{-6} \mathrm{MeV}^{-2}$. RPA results are indicated by bars and experimental data are represented by hatched areas. 
interaction Eq. (11) provides a reasonable fit to the experimental data. The baryon spectra presented here thus confirm the results for the meson sector. In both of these calculations the coupling constant $G$ enters as a parameter. In an NJL model based on a self-consistent treatment of the ground state the dynamics is governed by the dimensionless parameter $G \Lambda^{2}$. Due to the lack of self-consistency in our approach the single-particle cut-off $\Lambda$ is a priori not defined. However, we can view the energy of the lowest-lying hole state included in the RPA basis as a rough estimate of the 4-momentum scale under consideration. With this assumption we obtain $G \Lambda^{2} \approx 15$, compared to $G \Lambda^{2}=10.51$ of Ref. [7]. Considering the approximate fashion of such a comparison we conclude that the same coupling constant can be used to describe the baryon and the meson sector. A natural extension of our investigations with a simple prototype interaction would be to consider the most general chiral interaction with independent coupling strengths $G_{s}$ and $G_{v}$ for the scalar-pseudoscalar and the vector-axialvector channels. This could improve the description of the excitation spectrum.

We want to emphasize that $q \bar{q}$ admixtures to the wavefunctions are of considerable importance in the RPA spectra. Although the valence energy obtained with the harmonicoscillator potential is quite high the $p h$ basis contains more than twice as many excitations of the Dirac sea as valence excitations. Clearly the lowest-lying and thus most important states are the valence excitations. In some cases, however, certain $q \bar{q}$ amplitudes are of comparable order of magnitude. Thus in spite of the large energy gap the Dirac sea does not decouple.

The structure of the excitation spectrum is also sensitive to the choice of the mean field. In comparison to the MIT-bag results the transition of the levels to lower energies is smoothed down, as seen in Fig. 8. This is due to the relatively soft mean-field $m(r)=c r^{\alpha}$ with $\alpha=2$. This smoother transition extends the range of coupling constants where RPA effects are important and results in a simultaneous shift of excitation energies in several channels when the $p h$ interaction is switched on. However, an even softer parametrization $\alpha=1$ could provide a suitable ground-state configuration only when the size of the quark core is further reduced, as already pointed out in the previous chapter.

Although already a this stage a satisfactory description of the baryon spectrum has been achieved, a few shortcomings of our approach become apparent. In the $\left(\frac{1}{2}^{+}, \frac{1}{2}\right)$ channel the assumptions underlying the RPA approach rapidly break down due to an overestimation of ground-state correlations. In the $T=\frac{3}{2}$ channels the "phase transition" occurs at slightly higher values of $G$. Initially the energy of the $\Delta$ states with lowest angular momentum is slightly increased. A shift of the $\Delta$ states towards higher energies is expected from a color-current interaction. The most prominent example of the effects of a gluon-exchange force on the baryon spectrum has perhaps been given in the context of the MIT-bag model [42]. At higher coupling constants, however, this trend is overshadowed by rapidly growing ground-state correlations.

We expect that with a refined model for the ground state including dynamical correlations the "phase transition" is smoothed down. Within such a scenario the $T=\frac{3}{2}$ states could be lifted up more significantly, thus allowing to employ a model with a slightly bigger quark core. Nevertheless our calculations already indicate that the nucleon might 
contain a comparatively small quark core. The excitation spectrum appears to be closely connected to the dynamics of that quark core. It seems to be a reasonable approximation to account for the meson cloud only in an implicit manner, as inherent in a Dirac-RPA approach.

\section{Summary, Conclusion and Outlook}

The aim of this work was a phenomenological description of the nucleon excitation spectrum in the framework of the Dirac-RPA scheme developed in part I of this paper.

As an attempt towards a self-consistent treatment of the mean-field configuration entering the RPA scheme we have considered the Dirac-Hartree-Fock problem for a rotationally and isospin invariant ground-state density. For this purpose the Nambu-Jona-Lasinio model was used, which is known to possess solitonic solutions in the $B=1$ sector.

In a calculation with the most general form of a self-energy which is admissible within our treatment of symmetries the chiral condensate of the NJL model could be reproduced with high accuracy. The vacuum was found to be a translationally invariant state, thus supporting the assumptions which usually enter the Dyson-Schwinger equation for the vacuum self-energy.

The $B=1$ system, however, did not emerge as a solitonic object due to the unbound valence quarks. We conclude that the pseudoscalar field, which does not contribute in our description for reasons of symmetry, is of major importance for the formation of the soliton found in other approaches [4, 5]. The inclusion of a vector potential does not cure the problem, as expected from the repulsive character of the interaction. The results indicate that the existence of the soliton does strongly depend on the grand-spin approach. In order to allow for an application of the tensor-RPA method and to construct a simple confinement mechanism we have discussed a class of phenomenological models for the nucleon ground state. In these descriptions confinement is enforced by an asymptotically rising scalar potential.

We have solved the tensor RPA equation for different combinations of mean-field potentials and $p h$ interactions with focus on the positive-parity channels which are free of spurious admixtures. It was found that by inclusion of a residual interaction the excitation energies are lowered in most cases. With increasing interaction strength strong ground-state correlations develop. For a comparatively low valence energy these correlations predominantly originate from the Dirac sea.

Using a standard MIT-bag configuration with a nucleon rms of $0.71 \mathrm{fm}$ the experimental data for the excitation energies cannot be reproduced. The origin for this bad agreement lies in the high density of excited states which is even increased when the $p h$ interaction is switched on.

With regard to recent results obtained by Glozman and Riska 15 we have also studied the problem of an inversion of positive- and negative- parity states. It was found that in a relativistic TD approximation the lowest positive- and negative-parity states become inverted, whereas due to a strong coupling to ground-state correlations no such inversion is seen in the corresponding RPA calculation. 
In view of reproducing the experimental data for positive-parity baryons we have considered a quark core with a size of $\approx 0.3 \mathrm{fm}$. At the same time the rigid MIT-bag potential was replaced by a softer harmonic-oscillator potential. By these means the baryon spectrum could be reproduced fairly well, given that a local color-current interaction is chosen, with a coupling strength comparable to that of earlier work [7]. With a local $\sigma-\boldsymbol{\pi}$ interaction no comparable description of the baryon spectrum could be achieved.

Despite of these successes some problems related to the simplified description of the ground state remain. The RPA "phase transition" due to an overestimation of ground-state correlations effects a rapid breakdown of our method when the coupling constant is increased above a critical value. By inclusion of dynamical correlations in the ground-state wavefunction in an explicit fashion these difficulties could be overcome. Since such a task is quite involved, the corresponding refinements are left for future investigations.

Besides that two other issues clearly deserve a closer inspection. On the one hand we have made no attempt to improve the agreement with experimental data by finetuning the independent coupling constants $G_{s}$ and $G_{v}$ of a generalized chiral interaction. On the other hand it is necessary to consider transition matrix elements in order to compute the excitation probabilities of the various states in a $\pi N$ scattering experiment. On such a basis one might be able to eliminate certain states which are unobservable due to their reduced transition strength. One will also be able to compute the partial widths for, e. g., a decay into a $N \pi$ final state.

\section{References}

[1] S. Hardt, J. Geiss, H. Lenske and U. Mosel, Nucl. Phys. A, in press

[2] A. Abada, R. Alkofer, H. Reinhardt and H. Weigel, Nucl. Phys. A593 (1995), 488

[3] C. Schüren, F. Döring, E. Ruiz Arriola and K. Goeke, Nucl. Phys. A565 (1993), 687

[4] R. Alkofer, H. Reinhardt and H. Weigel, Phys. Rep. 265 (1996), 139

[5] C.V. Christov, A. Blotz, H.C. Kim, P. Pobylitsa, T. Watabe, T. Meissner, E. Ruiz Arriola and K. Goeke, Prog. Part. Nucl. Phys. 37 (1996) (in print)

[6] A. Buck and H. Reinhardt, Phys. Lett. B356 (1995), 168

[7] S. Klimt, M. Lutz, U. Vogl and W. Weise, Nucl. Phys. A516 (1990), 429

[8] N. Isgur and G. Karl, Phys. Rev. D18 (1978), 4187

[9] A. Umnikov and F. Khanna, Int. J. Mod. Phys. A11 (1996), 3935

[10] G. Fai, R. Perry and L. Wilets, Phys. Lett. B208 (1988), 1

[11] M.R. Frank and P.C. Tandy, Phys. Rev. C46 (1992), 338 
[12] M. Buballa and S. Krewald, Phys. Lett. B294 (1992), 19

[13] R.D. Bowler and M.C. Birse, Nucl. Phys. A582 (1995), 655

[14] K. Langfeld and M. Rho, Nucl. Phys. A596 (1996), 451

[15] L.Y. Glozman and D.O. Riska, Phys. Rep. 268 (1996), 263

[16] U. Vogl and W. Weise, Prog. Part. Nucl. Phys. 27 (1991), 195

[17] C.V. Christov, K. Goeke and M. Polyakov, "NJL Model with Vector Coupling vs. Phenomenology", LANL hep-ph/9501383

[18] K. Yamawaki and V.I. Zakharov, "Extended NJL Model vs. QCD Sum Rules", LANL hep-ph/9406373

[19] Y. Nambu and G. Jona-Lasinio, Phys. Rev. 122 (1961), 345

[20] J.J. Sakurai, Advanced Quantum Mechanics, Addison-Wesley, Reading, Ma. 1967

[21] R. Alkofer, H. Reinhardt, J. Schlienz and H. Weigel, Z. Phys. A354 (1996), 481

[22] P. Sieber, T. Meissner, F. Grümmer and K. Goeke, Nucl. Phys. A547 (1992), 459

[23] T. Watabe and H. Toki, Progr. Theor. Phys. 87 (1992), 651

[24] J. Schlienz, H. Weigel, H. Reinhardt and R. Alkofer, Phys. Lett. B315 (1993), 6

[25] T. Meissner and K. Goeke, Nucl. Phys. A524 (1991), 719

[26] D. Faiman and A.W. Hendry, Phys. Rev. 173 (1968), 1720

[27] V. Gillet, A.M. Green and E.A. Sanderson, Nucl. Phys. 88 (1966), 321

[28] H.A. Mavromatis, W. Markiewicz and A.M. Green, Nucl. Phys. A90 (1967), 101

[29] A. Chodos, R. Jaffe, K. Johnson, C. Thorn and V. Weisskopf, Phys. Rev. D9 (1974), 3471

[30] P. Schuck and S. Ethofer, Nucl. Phys. A212 (1973), 269

[31] J. Orear, Phys. Rev. D18 (1978), 2484

[32] G.E. Brown, M. Rho and W. Weise, Nucl. Phys. A454 (1986), 669

[33] C.L. Critchfield, J. Math. Phys. 17 (1976), 261

[34] P. Ring and P. Schuck, The Nuclear Many Body Problem, Springer, New York 1980

[35] J.-L. Dethier, R. Goldflam, E.M. Henley and L. Wilets, Phys. Rev. D27 (1983), 2191 
[36] G.E. Brown and M. Rho, Phys. Lett. B82 (1979), 177

[37] P.J. Mulders, Phys. Rev. D30 (1984), 1073

[38] J. Sucher, Phys. Rev. D51 (1995), 5965

[39] B.K. Bhaduri, Models of the Nucleon, Addison-Wesley, Redwood City 1988

[40] R. Bijker, F. Iachello and A. Leviathan, Ann. Phys. (NY) 236 (1994), 69

[41] U. Vogl, M. Lutz, S. Klimt and W. Weise, Nucl. Phys. A516 (1990), 469

[42] T.A. DeGrand and R.L. Jaffe, Ann. Phys. (NY) 100 (1976), 425

[43] D. Strottman, Phys. Rev. D20 (1979), 748

[44] H. Leutwyler, Ann. Phys. (N.Y.) 235 (1994), 165

[45] J.F. Donoghue, E. Golowich and B.R. Holstein, Dynamics of the Standard Model, Cambridge University Press, Cambridge 1992

[46] T.H.R. Skyrme, Proc. R. Soc. London A260 (1961), 127

[47] R. Alkofer and H. Reinhardt, Z. Phys. A343 (1992), 79

[48] D. Diakonov, "Chiral Symmetry Breaking by Instantons", LANL hep-ph/9602375

[49] L. Landau and E. Lifshitz, Quantum Mechanics, Pergamon Press, Oxford 1977

[50] J. Geiss, H. Lenske and U. Mosel, Z. Phys. A356 (1996), 99

[51] L. Glozman, Z. Papp and W. Plessas, Phys. Lett. B381 (1996), 311

[52] T. Ericson and W. Weise, Pions and Nuclei, Calendron Press, Oxford 1988

[53] Particle Data Group, Phys. Rev. D54 (1996), 1 